\title{
Charnoly body as a novel biomarker of nutritional stress in Alzheimer's Disease
}

\section{Sushil Sharma, Joseph Choga, Vineet Gupta, Pearl Doghor, Ankur Chauhan, Fredy Kalala, Alison Foor, Christopher Wright, James Renteria, Krystel Elliott- Theberge, Shubhra Mathur}

Saint James School of Medicine, Cane Hall, St Vincent, St Vincent and the Grenadines

Corresponding author: Sushil Sharma, PhD, Professor of Pharmacology, Saint James School of Medicine, Cane Hall, St Vincent, St Vincent and the Grenadines

Submission Date: April 18, 2016, Accepted Date: June 20, 2016, Publication Date: June 29, 2016

\begin{abstract}
Background: The Charnoly body (CB) was discovered as a universal biomarker of cell injury in the developing undernourished rat cerebellar Purkinje neurons and in the intrauterine Domoic acid and Kainic acid-exposed mice hippocampus and hypothalamic neurons. The incidence of $\mathrm{CB}$ increased with the severity of nutritional and environmental neurotoxic insult.
\end{abstract}

Purpose: We proposed that stress (nutritional and environmental) induced cortisol release augments, whereas metallothioneins (MTs), insulin-like growth factor (IGF-1), and brain-derived neurotropic factor (BDNF) inhibit $\mathrm{CB}$ formation to prevent progressive neurodegeneration, early morbidity, and mortality in Alzheimer's disease (AD).

Results: CB is a pre-apoptotic biomarker of compromised mitochondrial bioenergetics, formed in the most vulnerable cell in response to nutritional stress, intrauterine infection, environmental toxins, and/or drug abuse due to free radical overproduction and mitochondrial genome downregulation. It appears as a pleomorphic, electron-dense multi-lamellar, quasi-crystalline stack of degenerated mitochondrial membranes in highly susceptible neurons and may be induced by microbial infection. CB formation was accompanied with stunted neuritogenesis in the aging mitochondrial genome knock out $\left(\mathrm{RhO}_{\text {mgko }}\right)$ human dopaminergic (SK-N-SH, SHS-Y-5Y) neurons due to down-regulation of ubiquinone NADH oxidoreductase (complex-1). Transfection of $\mathrm{RhO}_{\text {mgko }}$ neurons with ubiquinone $\mathrm{NADH}$ oxidoreductase (complex-1) gene and $\mathrm{CoQ}_{10}$, inhibited $\mathrm{CB}$ formation and augmented neuritogenesis, as confirmed in $\alpha$-synucleinmetallothioneins triple knock out and weaver mutant mice. CB formation was attenuated in MTsover-expressing weaver mutant mice. 
Findings: Accumulation of $\mathrm{CB}$ at the junction of axon hillock impairs axoplasmic transport of enzymes, neurotransmitters, hormones, neurotropic factors (NGF, BDNF), and mitochondria at the synaptic terminals cause cognitive impairment, early morbidity, and mortality. Nonspecific induction of CB causes alopecia, myelosuppression, and GIT symptoms in multi-drug-resistant malignancies. Antioxidants and MTs inhibit CB formation as free radical scavengers by zincmediated transcriptional regulation of genes involved in growth, proliferation, differentiation, and development. Consequently, drugs may be developed to prevent $\mathrm{CB}$ formation and/or enhance charnolophagy as a basic molecular mechanism of intracellular detoxification to avert cognitive impairments in AD.

Conclusion: Brain regional monoamine oxidase-specific $\mathrm{CBs}$ can be detected by ${ }^{11} \mathrm{C}$ or ${ }^{18} \mathrm{~F}$ labeled MAO-A or MAO-B inhibitors in vivo, in addition to ${ }^{18} \mathrm{FdG}$-PET neuroimaging to quantitatively assess and improve the mitochondrial bioenergetics in AD.

\section{BACKGROUND}

The Charnoly body (CB) was discovered for the first time in the developing undernourished rat cerebellar Purkinje neurons due to nutritional stress-induced free radical overproduction [1, 2]. Selective neurodegeneration in the hippocampal CA-3 and dentate gyrus region was accompanied with cognitive impairment, CB formation, and apoptosis in the intra-uterine excitoneurotoxins, Kainic acid and Domoic acid-exposed CD-1 mice, as observed in the autopsy brain samples of AD patients [3, 4]. Intra-hippocampal microinjections of Domoic acid induced severe seizure discharge activity of prolonged duration in the adult mice. Domoic acid-induced seizure discharge activity was suppressed by specific $5-\mathrm{HT}_{1 \mathrm{~A}}$ receptor agonist, 8-OH-DPAT, and was augmented by a specific 5-HT $1 \mathrm{~A}$ antagonist, Spiroxatrine, as confirmed by computerized EEG analyses [5]. Subsequently, we reported CB formation and its pathophysiological significance in various cellular and animal models of PD [6], AD [7], vascular dementia [8], fetal alcohol syndrome [9, 10], and in human and animal models of chronic drug addiction [11]. We reported that low molecular weight metal (zinc-binding), cysteine-rich proteins, metallothioneins (MTs) confer therapeutic potential as anti-inflammatory and anti-apoptotic-agents in polysubstance abuse [12, 13] and in PD [14, 15]. Furthermore, we reported the therapeutic potential of MTs in the aging brain as potent free radical scavengers and CB antagonists in obesity [16]. MTs prevent the induction of CBs (pre-apoptotic biomarker of compromised mitochondrial bioenergetics) by serving as free radical scavengers. MTs also confer neuroprotection by zinc-mediated transcriptional regulation of genes involved in growth, proliferation, differentiation, and development. MTs (particularly MT-3) are significantly down-regulated in AD and aging brain. We confirmed our original hypothesis that MTs confer ubiquinone (CoQ $\left.{ }_{10}\right)$-mediated neuroprotection in progressive neurodegenerative diseases such as $\mathrm{PD}, \mathrm{AD}$, and chronic drug addiction by employing MTs gene manipulated cultured human dopaminergic (SK-N-SH, SH-S$\mathrm{Y} 5 \mathrm{Y})$ neurons in vitro and in control wildtype, metallothionein double gene knock out $\left(\mathrm{MT}_{\mathrm{dko}}\right)$, metallothioneins transgenic ( $\mathrm{MT}_{\text {trans }}$ ), $\alpha$-synuclein-metallothionein triple knock out ( $\alpha$-syn- 
$\mathrm{MT}_{\mathrm{tko}}$ ), and MTs-over-expressed weaver mutant (wv/wv-MTs) mice in vivo. Brain regional $\mathrm{CoQ}_{10}$ levels were significantly high in $\mathrm{MT}_{\text {trans }}$ mice as compared to control ${ }_{\mathrm{wt}}$ and $\mathrm{MT}_{\mathrm{dko}}$ mice, strengthening our original hypothesis. Indeed, $\mathrm{MT}_{\text {trans }}$ mice are lean and agile whereas $\mathrm{MT}_{\mathrm{dko}}$ mice are lethargic and mildly obese, further confirming that MTs provide mitochondrial neuroprotection by preventing $\mathrm{CB}$ formation in the aging brain [17].

$\mathrm{CB}$ is a highly unstable, pleomorphic, multi-lamellar, electron-dense membrane stack formed due to mitochondrial degeneration in response to nutritional stress and/or toxic exposure [18]. Free radical-induced compromised mitochondrial bioenergetics triggers CB formation in the most vulnerable cell. CB formation occurs as a primary and early event in the etiopathogenesis of intra-neuronal inclusions in various neurodegenerative diseases. Free radicalinduced mitochondrial $\Delta \Psi$ collapse triggers oxidation of mtDNA to cause $8-\mathrm{OH}, 2 \mathrm{dG}$ synthesis and $\mathrm{CB}$ formation, which is the initial stage in the induction of progressive neurodegenerative diseases. Non-specific induction of CB formation causes GIT stress, myelosuppression, and alopecia in multi-drug resistant malignancies and major depressive disorders (MDDs) [19]. Recently, we reported that depending on the type of neurons involved, MAO-A or MAO-B specific CBs may be formed in the soma, as well as in the synaptic regions of DA-ergic, 5HTergic, NE-ergic, GABAergic or other neurons. Consequently, novel drugs may be developed to prevent and/or eradicate MAO-A or MAO-B specific $\mathrm{CB}$ formation to provide neuroprotection [19]. In particular, charnolophagy is the most efficient and intricate basic molecular mechanism of intracellular detoxification during acute phase of neurotoxicity and/or nutritional insult in the developing or aging brain. $\mathrm{CB}$ sequestration during chronic phase releases MAOs, as well as cytochrome-C to not only cause depletion of synaptic NE, 5-HT and DA, but also cause apoptosis to enhance progressive neurodegeneration. MAOIs such as Selegiline and Rasagiline augment brain regional MTs to provide neuroprotection by inhibiting $\mathrm{CB}$ formation and by serving as potent free radical scavengers. Therefore, novel MAOIs or other mitochondrially-targeted drugs may be developed to prevent tissue-specific CB formation, enhance charnolophagy and/or prevent $\mathrm{CB}$ sequestration for effective and safe treatment of PD, $\mathrm{AD}$, drug addiction, and MDDs, and vice versa for the personalized treatment of multidrug resistant malignancies with minimum or no adverse effects [19].

In general, free radicals ( ${ }^{*} \mathrm{OH}, * \mathrm{NO}$ )-induced $\alpha$-Synuclein Index triggers mitochondrial degeneration to enhance $\mathrm{CB}$ formation and eventually Lewy body formation, implicated in the degeneration of DA-ergic neuron in PD and senile (amyloid body) plaque formation in AD. Similarly, brain region-specific protein nitration is involved in CB formation and eventually accumulation of intra-neuronal inclusions in various neurodegenerative disorders. Accumulation of $\mathrm{CBs}$ at the junction of axon hillock can impair normal axoplasmic flow of various ions, enzymes, neurotropic factors (BDNF, IGF-1, NGF), and mitochondria to cause abnormal axoplasmic flow and hence degeneration of synaptic terminals to cause abnormal synaptic neurotransmission and eventually cognitive impairments in AD and aging [20].

This review provides a recent update on the molecular mechanism of $\mathrm{CB}$ pathogenesis in $\mathrm{AD}$ and its prevention and/or inhibition by antioxidants derived from functional foods. The 
distinct advantage of antioxidants is that they can easily pass through blood brain barrier without any adverse effect and can efficiently scavenge devastating free radicals, generated as a byproduct of mitochondrial oxidative phosphorylation during ATP synthesis. Furthermore, we have highlighted the therapeutic potential of MTs as CB antagonists in AD prevention and cortisol as a potent agonist of hippocampal CB formation in AD progression. We have proposed disease-specific natural or synthetic $\mathrm{CB}$ antagonists and $\mathrm{CB}$ sequestration inhibitors in the effective clinical management of progressive neurodegenerative diseases such as AD and cancer stem cell-specific CB inducers and/or CB sequestrants for the effective clinical management of multi-drug-resistant malignancies.

\section{CB as a Universal Vs Disease-Specific Biomarker}

We described CB as a universal biomarker of cell injury in one of our recent publications [6]. Indeed, a cell cannot survive without mitochondria. Depending on the metabolic demand and the function, a cell may have between 120-1000 mitochondria of different size, shape, geometry, and topography. Any physicochemical injury, malnutrition, depletion of antioxidants such as MTs, and/or infection can induce free radical overproduction, lipid peroxidation, mitochondrial DNA damage, and degeneration of mitochondrial membranes, leading to $\mathrm{CB}$ formation in a highly vulnerable cell. Thus, CB formation due to nutritional (functional foods) and/or antioxidants deficiency is classified as a universal biomarker of cell injury, whereas the disease-specific CB formation is cell-specific and occurs primarily in response to a particular illness such as neurodegenerative diseases (PD, AD, MDD, chronic drug addiction), cardiovascular diseases, and cancer. The clinical significance of disease-specific $\mathrm{CB}$ formation is that it can be utilized for the development of novel drugs against a particular disease. In this respect, disease-specific $\mathrm{CB}$ inhibitors and $\mathrm{CB}$ sequestration inhibitors will be therapeutically useful for the clinical management of cardiovascular and neurodegenerative diseases, whereas $\mathrm{CB}$ inducers and/or CB sequestrants will be therapeutically significant for the clinical management of multi-drugresistant malignancies [19].

\section{MTs as CB Antagonists}

We reported that MTs are low molecular weight, cysteine-rich, zinc binding proteins, and are induced as a protective mechanism in the most vulnerable cell in response to nutritional and/or environmental neurotoxic insult. Free radicals ( $\left.{ }^{*} \mathrm{OH}, * \mathrm{NO}\right)$ generated as a byproduct during oxidative phosphorylation are readily scavenged by SOD, catalase, and glutathione. However, during severe nutritional stress or toxicity, induced MTs are translocated in the mitochondria and in the nucleus to protect the mtDNA as well as nuclear DNA respectively. MTs are almost 30 times more potent as compared to glutathione and serve as free radical scavengers to prevent $\mathrm{CB}$ formation during oxidative and nitrative stress of nutritional, environmental, and aging. Therefore, pharmacological interventions to enhance MTs can provide neuroprotection in progressive neurodegenerative diseases by inhibiting free radical-induced $\mathrm{CB}$ formation in PD, AD, Drug addiction, MDD, and schizophrenia [16] as Figure 1 illustrates. 


\section{MTs Inhibit CB Formation to Prevent AD}

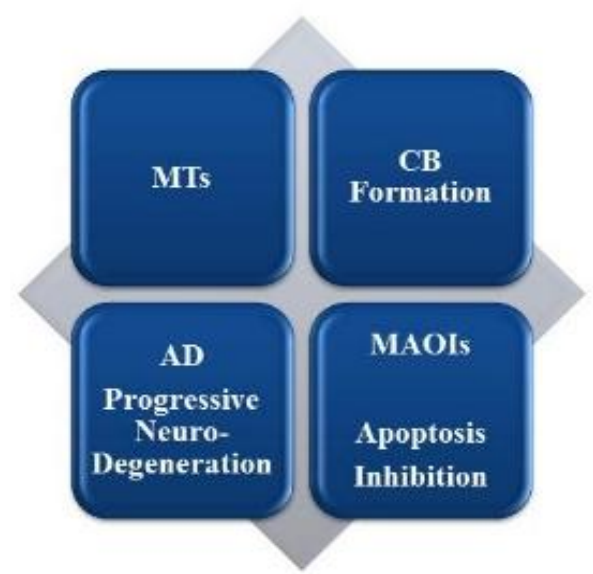

Figuere 1. A schematic flow diagram illustrating MTs-mediated inhibition of CB formation. MTs inhibit CB formation by serving as free radical scavengers and vice versa. MAOIs such as Selegiline and Rasagiline inhibit apoptosis by preventing CB formation. MAOIs induce MTs to prevent CB-mediated apoptosis to provide neuroprotection in AD.

\section{Detailed Description of $\mathrm{CB}$}

We reported $\mathrm{CB}$ as a universal biomarker of cell injury which is induced in response to genetic, environmental, and/or toxic insult due to free radical-mediated damage of mitochondrial membranes in a highly vulnerable cell [21]. Free radicals are highly reactive, ultra-short-lived $\left(\mathrm{t}_{1 / 2}: 10^{-13}-10^{-14} \mathrm{Sec}\right)$ reactive oxygen species $(* \mathrm{OH}, * \mathrm{NO})$ and cause lipid peroxidation, which triggers structural and functional breakdown of polyunsaturated fatty acids in the plasma membranes to cause neuronal cell damage through $\mathrm{CB}$ formation. $\mathrm{CB}$ appears as a highly unstable, pleomorphic, electron-dense, multi-lamellar membrane stacks in the perinuclear region or adjacent to the desmosomes or degenerating membranes as a hallmark of compromised mitochondrial bioenergetics. The aggregation of $\mathrm{CB}$ at the junction of axon hillock can impair normal axoplasmic flow of various ions, neurotransmitters, hormones, neurotrophic growth factors, and mitochondria to cause cognitive impairment. $\mathrm{CB}$ formation in the hippocampal region may cause $\mathrm{AD}$, in the NS-DA-ergic region may cause $\mathrm{PD}$, and in the mediobasal hypothalamic region may cause bulimia. CB formation may also be utilized as an early and sensitive biomarker of neurodegeneration in MDDs. Nonspecific induction of CB formation in multidrug-resistant malignancies causes GIT distress, myelosuppression, and alopecia [18]. Consequently, novel CB antagonists as synthetic or natural antioxidants in functional foods may be developed to prevent earlier phase of disease progression, charnolophagy agonists during intermediate phase as intracellular detoxifiers, and $\mathrm{CB}$ sequestration inhibitors during chronic phase as novel therapeutic agents in neuro-degenerative and cardiovascular diseases, and vice versa for the eradication of multi-drug resistant malignancies with minimum or no adverse effects. We reported that during chronic phase, lysosomal resistant CB develops. Hence, CB sequestration during chronic phase may induce neurodegeneration due to the uncontrolled 
intracellular release of cytochrome $\mathrm{C}$, iron, caspase-3, and free radicals, which can be prevented by developing novel CB sequestration antagonists [18].

\section{CB Life Cycle}

We primarily discovered the four major phases of CB life cycle using light microscopy, digital fluorescence microscopy, scanning electron microscopy (SEM), and transmission electron microscopic (TEM) analyses of developing undernourished rat cerebellar Purkinje neurons [21]. Purkinje neurons develop postnatally in the rat brain and are highly susceptible to the deleterious consequences of nutritional and environmental neurotoxic insult. The four major phases of $\mathrm{CB}$ life cycle are: (i) Origin, (ii) Development, (iii) Maturation, (iv) Degradation. At the ultrastructural level, CB formation occurs in the most vulnerable developing Purkinje neurons due to nutritional stress and is accompanied with enhanced production of free radicals, which induce lipid peroxidation of the mitochondrial membranes to enhance neurodegeneration. The early biomarkers of $\mathrm{CB}$ can be determined by estimating mitochondrial membrane potential $(\Delta \Psi)$ collapse and $8-\mathrm{OH}, 2 \mathrm{dG}$ synthesis by digital fluorescence microscopic analysis and by performing enzyme linked immunosorbent assay (ELISA). The aggregation and condensation of the degenerated mitochondrial membranes involved in $\mathrm{CB}$ formation can also be determined by SEM, atomic force microscopy, and confocal microscopy. Although confocal microscopic analysis provides in-vivo information regarding CB formation in the perinuclear region, resolution remains a significant challenge. A detailed ultrastructural analysis of development, maturation, and degradation by lysosomes (charnolophagy) at higher resolution can be accomplished by sequential TEM analyses.

CB formation occurs primarily based on the following basic principle of molecular biology: "Similar molecules associate, whereas dissimilar molecules dissociate". Although molecular association of degenerated mitochondrial membranes results in $\mathrm{CB}$ formation, these multilamellar, electron dense membrane stacks are unable to synthesize ATP by oxidative phosphorylation, store $\mathrm{Ca}^{2+}$, synthesize steroid hormones, and are unable to perform several diversified functions of normal healthy mitochondria. These nonfunctional, degenerated, and aggregated mitochondrial membranes (named as $\mathrm{CBs}$ ) are usually detected as penta or heptalamellar electron dense quasi-crystalline structures, and can impair normal axoplasmic flow of various ions, neurotransmitters, hormones, growth factors, and mitochondria at the synaptic terminals if they are accumulated at the junction of axon hillock to cause cognitive impairments, as noticed in PD, AD, MDD, and chronic drug addiction [18-21].

In general, $\mathrm{CB}$ formation in a highly vulnerable cell is associated with compromised mitochondrial bioenergetics, TSPO delocalization, $\mathrm{Ca}^{2+}$ dyshomeostasis, accumulation of cholesterol, loss of steroid hormones synthesis, down-regulation of mitochondrial genome, partial or complete loss of mitochondrial function, and predisposition of the cell to apoptosis. The CB formation in the developing undernourished Purkinje neurons occurred concomitantly with significantly increased latency of electrophysiological response to peripheral electrical stimulation, reduced amplitude and frequency of firing, and prolonged after-hyperpolarization duration, due to increase in $\left[\mathrm{Ca}^{2+}\right]_{\mathrm{i}}$. The frequency of $\mathrm{CB}$ formation increased as a function of nutritional stress and reduced during nutritional rehabilitation. Virtually all $\mathrm{CBs}$ were phagocytosed during nutritional rehabilitation by lysosomes as a basic molecular mechanism of 
intracellular detoxification. The phagocytosis of mature CBs by lysosomes was defined as charnolophagy. We observed that in serum-deprived cultured vascular smooth muscle cells, lysosomal resistant CBs sequester to release cytochrome C, apoptosis-inducing factor (AIF), iron, and Bax in vitro. These changes at the molecular level may induce atherosclerotic plaque rupture in cardiovascular diseases $[18,21]$.

\section{Hypothesis}

Consequently, we now propose that stress (nutritional and environmental) induced cortisol releases augments, whereas MTs, IGF-1, and BDNF inhibit hippocampal CB formation to prevent progressive neurodegeneration, thereby preventing early morbidity and mortality in AD.

\section{$M A O-A$ and MAO-B Specific $C B$}

The brain regional distribution of MAOs varies depending on the type of mitochondria in the neurons. Although dopamine can be oxidized equally by both MAO-A and MAO-B, the striatal concentration of MAO-B is very high in the striatum, which is the primary rationale of prescribing MAO-B-specific inhibitors including Selegiline, Rasagiline to delay the requirement of L-DOPA therapy in PD patients. Similarly, MAO-A rich mitochondria are present in the periaqueductal gray and dorsal raphe (these brain regions are primarily 5-HTergic) and are involved in the etio-pathogenesis of MDDs. MAO-A inhibitors, as well as specific serotonin reuptake inhibitors, are prescribed for the clinical management of mood swings (depression and anxiety and pain control). Thus, these regions are highly rich in MAO-A specific CB formation and require the development of novel drugs which could specifically inhibit MAO-A specific CB formation. We reported that specific monoamine oxidase-B inhibitors, Selegiline and Rasagiline provide mitochondrial neuroprotection through MTs-mediated CB inhibition by serving as potent free radical scavengers [18]. Several other antioxidants including: Melatonin, Sirtuins, Resveratrol, and $\mathrm{CoQ}_{10}$ present in functional foods may also serve as $\mathrm{CB}$ inhibitors to provide neuroprotection. Exactly similar basic molecular mechanism of mitochondrial neuroprotection might be conferred by MAOIs for better patient care, treatment of chronic diseases, promotion of health, and better quality of life by preventing disease-specific $\mathrm{CB}$ formation. Based on two types of MAOs, localized on the outer mitochondrial membranes, two types of CBs were proposed: (a) MAOI-A-Specific CBs and (b) MAOI-B-Specific CBs as novel drug discovery targets for the personalized treatment of $\mathrm{PD}, \mathrm{AD}$, chronic drug addiction, post-traumatic stress disorder (PTSD), and multi-drug resistant MDDs, as unique platform for future research and development of novel therapeutic drugs. MAO-specific CB antagonists will have superior pharmacological profile, enhanced margin of safety, maximum therapeutic index, and minimum or no adverse effects [19, 20,21].

\section{${ }^{18}$ F-DOPA and ${ }^{18}$ FdG Neuroimaging in Experimental Model of Neurodegeneration}

Control $_{\mathrm{wt}}, \mathrm{MT}_{\mathrm{dko}}, \mathrm{MT}_{\text {trans }}, \alpha-\mathrm{Syn}_{\mathrm{ko}}, \alpha-\mathrm{Syn}-\mathrm{MT}_{\mathrm{tko}}, \mathrm{wv} / \mathrm{wv}$, and wv/wv-MTs mice were raised to confirm the therapeutic potential of MTs as CB inhibitors. MicroPET imaging was performed employing Siemens Medical Solutions microPET imaging system, equipped with microPET Manager for the data acquisition and AsiPro for 3D image reconstruction as described in our several publications [21-25]. MicroPET imaging of ${ }^{18} \mathrm{FdG}$ in C57BL/6J mouse exhibited 
maximum localization in the $\mathrm{CNS}$, heart, lungs and the adipose tissue. ${ }^{18} \mathrm{FdG}(250 \mu \mathrm{Ci})$ was injected in the caudal vein after $350 \mathrm{mg} / \mathrm{kg}$, i.p Tribromoethane anesthesia. After $20 \mathrm{~min}$, the imaging data was acquired by microPET Manager and analyzed by Asi-Pro software. ${ }^{18} \mathrm{~F}$-DOPA $(250 \mu \mathrm{Ci})$ was injected in the caudal vein to acquire sequential in-vivo dynamic functional images. The image analyses revealed significantly reduced striatal ${ }^{18} \mathrm{~F}$-DOPA uptake in wv/wv mouse as compared to control and wv/+ mice [23]. Ethanol augmented Cocaine and METHinduced neurotoxicity in the CNS and other body parts such as heart and lungs of C57BL/6J mice. Imaging data were also acquired from C57BL/6J mice intoxicated with Cocaine, Cocaine+METH, and Cocaine+METH+Ethanol respectively for 21 days [26]. ${ }^{18}$ F-DOPA and ${ }^{18} \mathrm{FdG}$ were used as PET biomarkers to assess brain regional DA-ergic neurotransmission and mitochondrial bioenergetics, respectively. The striatal ${ }^{18} \mathrm{~F}$-DOPA uptake was significantly reduced in Cocaine-treated mice [27]. Co-administration of METH induced further reduction in the striatal ${ }^{18} \mathrm{~F}$-DOPA uptake. A severe reduction in the striatal ${ }^{18} \mathrm{~F}$-DOPA uptake was observed in mice intoxicated with Cocaine+METH, and Ethanol concomitantly, suggesting that Ethanol serves as a gateway to multiple drug abuse. Cocaine in combination with Ethanol, forms a highly toxic metabolite, Coco-ethylene, which induces severe loss of striatal DA-ergic and hippocampal cholinergic neurons. ${ }^{18} \mathrm{FdG}$ uptake was also significantly reduced in the myocardium and lungs as observed in the coronal and sagittal sections of these experimental models of multiple drug abuse [27]. These studies demonstrated that brain regional DA-ergic, as well as myocardial and pulmonary mitochondrial bioenergetics, are significantly compromised thereby causing early morbidity and mortality in multiple drug abuse. Recently, we reported that multiple drug abuse triggers progressive neurodegeneration due to free radical mediated CB formation, involved in apoptosis and pro-inflammatory cascade to cause cognitive impairment, early morbidity, and mortality, whereas antioxidants, such as MTs, prevent CB formation and progressive neurodegeneration by serving as potent free radical scavengers [13].

The cellular and molecular biology of CB is now well established as an early and sensitive biomarker for the clinical diagnosis and prognosis of PD, AD, and drug addiction. Described below are the distinct cellular, subcellular, and molecular events involved in CB pathogenesis and their prevention/inhibition in progressive neurodegenerative diseases including PD, AD, MDD, schizophrenia, and chronic drug addiction. Early detection of molecular events involved in CB pathogenesis can be helpful in preventing and/or treating these neurodegenerative diseases [29].

\section{8-OH, 2dG Synthesis}

As the mtDNA is not protected by histones and protamines, it can be readily oxidized as well as methylated. Although the DNA sequence remains unaltered during free radical-induced oxidative and nitrative stress, these epigenetic changes can significantly impact the health and well-being of an individual. The oxidation of the mtDNA generates $8-\mathrm{OH}, 2 \mathrm{dG}$, which is significantly increased in the plasma, serum, and urine samples of $\mathrm{PD}, \mathrm{AD}$, and aging subjects. By translocating in the nuclear region, $8-\mathrm{OH} 2 \mathrm{dG}$ can impair epigenetic changes required for the normal body functions and may induce impaired DNA methylation and histone acetylation as observed in chronic neurodegenerative diseases including $\mathrm{AD}, \mathrm{PD}$, and drug addiction. We developed multiple fluorochrome comet assay to detect $8-\mathrm{OH}, 2 \mathrm{dG}$ in the cultured human 
dopaminergic (SK-N-SH) neurons in response to overnight exposure of $\mathrm{MPP}^{+}(10 \mu \mathrm{M})$ by employing FITC-conjugated 8-OH, 2dG antibody [20].

It is now well established that hyper-methylation of the promoter region: of IGF-1 causes insulin-resistant type-2 diabetes, of BDNF causes major depression; of leptin causes obesity, and of VEGF causes stroke. In particular, chronic consumption of Ethanol can induce global hypomethylation and promote region hyper-methylation to cause hepato-encephalopathy. Additionally, Ethanol consumption during 3rd trimester of pregnancy may cause fetal alcohol syndrome (FAS) characterized by typical craniofacial abnormalities due to CB formation in the highly vulnerable cells of the developing embryo. Moreover, $8-\mathrm{OH}-2 \mathrm{dG}$ interferes with the normal function of microRNAs involved in various aspects of health and disease $[9,10]$.

\section{Epigenetic Changes as CB Modulators}

It is now well-established that epigenetic changes are implicated in health and disease. These changes occur due to methylation of DNA at the cytosine residue and histone acetylation at the lysine residues. Although the sequence of bases remains unchanged, these epigenetic changes can induce several physiological and biochemical alterations in the living system due to change in the basic conformation of DNA molecule. The mitochondrial DNA is a single-stranded uncoiled structure of $34 \mathrm{~Kb}$ size. It has only coding (exons) regions, no non-coding (introns) regions, and remains in a hostile environment of free radicals, generated as a byproduct of the oxidative phosphorylation during ATP synthesis. In contrast, the nuclear DNA is a doublestranded helical structure, with exons and introns, and remains protected by histones and protamines, which confer structural and functional stability to the nuclear DNA. The nuclear DNA also remains protected by nucleoplasm and a double-walled membrane which provide further protection. As a result, the nuclear DNA remains highly preserved from the deleterious attacks of free radicals.

In general, severe nutritional and/or environmental toxic stress may induce uncoiling of the double-stranded nuclear DNA to induce epigenetic changes. Histone acetylation occurs at the lysine residues and is inhibited by lysine deacetylases (LSDs: such as Sirtuins, Rutins) and impaired by Ethanol consumption. Methionine is converted to S-Adenosyl Methionine in the presence of ATP, synthesized by mitochondria. S-Adenosyl methionine translocates in the nuclear region to cause DNA methylation at the cytosine residue as a crucial epigenetic event in health and disease. Usually cytosine is converted to methyl cytosine and hydroxymethyl cytosine and can be detected by ELISA in the biological fluids such as saliva, serum, CSF, and urine samples of AD or other patients to evaluate early epigenetic changes in the DNA molecule. A precise and quantitative estimation of DNA methylation and histone acetylation can be made by genome-wide next generation sequencing (NGS). These epigenetic changes at the mtDNA and/or nuclear DNA level can modulate CB formation in a living system. A limited information is currently available in this direction. Further studies will provide the exact role of epigenetic changes in CB molecular pathogenesis and its functional significance in health and disease [21].

\section{a-Synuclein Index as a Sensitive Indicator of CB Formation}

$\alpha$-Synuclein is a synaptic protein and is involved in normal DA-ergic neurotransmission. During oxidative and nitrative stress, $\alpha$-Synuclein is converted to nitrated $\alpha$-Synuclein. The nitrated $\alpha$ - 
Synuclein can easily aggregate to form Lewy bodies in PD. We discovered $\alpha$-Synuclein Index (SI), which is a ratio of the native $\alpha$-Synuclein vs nitrated $\alpha$-Synuclein in $\mathrm{MPP}^{+}$-exposed cell culture model of PD. The SI can be estimated from the biological fluids including CSF, serum, plasma, and saliva as a sensitive diagnostic biomarker of $\mathrm{CB}$ formation and progressive neurodegeneration [20]. We estimated SI by performing double radio-immunoprecipitation of native and nitrated $\alpha$-Synuclein, and by immunofluorescence microscopic analyses in saline control and $\mathrm{MPP}^{+}$-treated cultured SK-N-SH cells. Currently, SI can be easily and efficiently determined by employing mass spectroscopic analyses, capillary electrophoresis, FRET analyses, and by microPET neuroimaging with ${ }^{13} \mathrm{~N}$-labeled $\alpha$-Synuclein as the double radioimmunoprecipitation method which we used in our original studies is relatively time consuming and cumbersome.

\section{Cortisol as a CB Inducer}

It is now well-established that diversified types of stress (social, physical, mental, economical, psychological, physiological) can induce increased release of cortisol from the adrenal cortex. Cortisol is a potent inducer of CB formation in the CNS. Excessive release of cortisol in circulation causes induction of glucocorticoid receptors in the hippocampal CA-3 and dentate gyrus neurons to enhance glutamate (particularly NMDA) receptors involved in long term potentiation (LTP) and memory consolidation. Enhanced release of cortisol also activates microglia, which release pro-inflammatory cytokines such as IL-6, TNF $\alpha$ and NFk $\beta$ to cause neuronal apoptosis through $\mathrm{CB}$ formation, which further augments neurodegeneration to eventually impact cognitive performance of an $\mathrm{AD}$ patient. There is now ample evidence to suggest that degeneration of hippocampal neurons in MDD occurs due to cortisol-induced BDNF down-regulation and microglial activation as a pro-inflammatory response. Increased circulating levels of cortisol causes neurodegeneration through microglial activation, which enhances free radicals overproduction as well as induction of pro-inflammatory cytokines to cause further neurodegeneration, whereas astrocytes augment BDNF synthesis and scavenge free radical to provide neuroprotection. We are currently estimating salivary cortisol and BDNF levels in our participants by utilizing a sensitive enzyme-linked immunosorbent assay (ELISA) to evaluate the nutritional status and mood to establish that elevated cortisol levels have deleterious effect on mood (depression and anxiety) as noticed frequently in $\mathrm{AD}$ patients.

\section{Cortisol-Induced Hippocampal CB Formation}

Increasing evidence suggests that stress-induced cortisol release sensitizes brain microglia and enhances pro-inflammatory cytokines in progressive neurodegenerative diseases including; PD, $\mathrm{AD}$, and MDDs [30-33]. Furthermore, chronic cortisol release causes microglial activation through free radical overproduction to trigger $\mathrm{CB}$ formation, involved in progressive neurodegeneration. Recently, we reported hippocampal CB formation as an early and sensitive pre-apoptotic biomarker of compromised mitochondrial bioenergetics in $\mathrm{PD}, \mathrm{AD}$ and depression [6]. We also described the basic molecular mechanism of hippocampal $\mathrm{CB}$ formation in response to chronic stress-induced cortisol release and IGF-1 and BDNF depletion in MDDs. Therefore, $\mathrm{CB}$ formation can serve as an excellent biomarker of nutritional stress in $\mathrm{AD}$ and other progressive neurodegenerative diseases [34, 35, 36]. 


\section{$\mathrm{RhO}_{\text {mgko }}$ Cells as Experimental Model of Aging}

The mitochondrial genome is highly susceptible to nutritional and environmental toxic insult as it is intron-less, low molecular weight $34 \mathrm{~Kb}$ single stranded macromolecule. The mitochondrial genome is downregulated in $\mathrm{PD}, \mathrm{AD}$, and other chronic neurodegenerative diseases. We prepared mitochondrial genomic knock out $\left(\mathrm{RhO}_{\text {mgko }}\right)$ human dopaminergic (SK-N-SH) neurons by using $5 \mathrm{ng} / \mathrm{l}$ ethidium bromide in the cultured Dulbecco's Modified Eagle's Medium (DMEM), high glucose and glutamine, and $\mathrm{NaHCO}_{3}$ for 8 weeks in culture. At this minimum concentration of ethidium bromide, the nuclear DNA remains structurally and functionally intact, whereas the ability of the mtDNA to generate ubiquinone-NADH-Oxidoreductase (Complex-1) is significantly compromised. As a result, $\mathrm{RhO}_{\text {mgko }}$ neurons become highly de-differentiated and their important subcellular events including; tubulinogenesis, myelinogenesis, axonogenesis, synaptogenesis, and neuritogenesis are significantly compromised. However, these cells do not lose their potential to divide. We authenticated $\mathrm{RhO}_{\text {mgko }}$ neurons by performing RT-PCR of complex-1 gene, which is specifically down-regulated and these cells synthesize significantly high amounts of 8-OH-2dG as compared normal aging cells. Additionally, these neurons have significantly reduced $\mathrm{CoQ}_{10}$, stunted growth, and loss of neuritogenesis. Transfection of $\mathrm{RhO}_{\text {mgko }}$ neurons with ubiquinone (NADH) oxidoreductase (Complex-1) gene 1 regained neuritogenesis and these neurons were differentiated to perform normal biochemical and physiological functions [17]. In addition to significant increase in $\mathrm{CoQ}_{10}$; complex-1 gene-transfected $\mathrm{RhO}_{\text {mgko }}$ neurons exhibited improved axonogenesis, tubulinogenesis, myelinogenesis, synaptogenesis, neuritogenesis, and elaborate neuro-circuitry, beyond the scope of this manuscript. These original findings suggest that the mitochondrial bio-energetics is significantly compromised in the aging brain to cause cognitive impairments in AD. Hence pharmacological and/or nonpharmacological approaches to maintain the structural and functional integrity of mtDNA and inhibition of free radical overproduction by functional foods could sustain the mitochondrial bioenergetics and inhibit $\mathrm{CB}$ formation involved in $\mathrm{AD}$ pathogenesis. Further studies in this direction will go a long way in the successful clinical management of $\mathrm{AD}$ and other diseases of aging.

\section{MTs Provide Ubiquinone-Mediated Neuroprotection by Inhibiting CB Formation}

We raised several colonies of control wild-type $(\mathrm{C} 57 \mathrm{BL} / 6 \mathrm{~J}$ mice), metallothionein double gene knock out $\left(\mathrm{MT}_{\mathrm{dko}}\right)$ mice, and metallothionein transgenic $\left(\mathrm{MT}_{\text {trans }}\right)$ mice and weaver mutant (wv/wv) mice to authenticate our original hypothesis that "MTs provide Ubiquinone- $\left(\mathrm{CoQ}_{10}\right)$ mediated neuroprotection by inhibiting CB formation". We estimated brain regional $\mathrm{CoQ}_{10}$ in these experimental genotypes. The striatal $\mathrm{CoQ}_{10}$ levels were significantly reduced in $\mathrm{MT}_{\mathrm{dko}}$ and $\mathrm{wv} / \mathrm{wv}$ mice as compared to control ${ }_{\text {wild type }}$ and $\mathrm{MT}_{\text {trans }}$ mice. The striatal $\mathrm{CoQ}_{10}$ levels were significantly reduced particularly in wv/wv mice exhibiting typical symptoms of body tremors, postural irregularities, and cognitive impairments [17]. Thus, we developed a highly sensitive method to estimate the negligible amounts of the striatal $\mathrm{CoQ}_{10}$ in these genetically rare animal model of multiple drug abuse [37]. We estimated CoQ homologues, neurotransmitters, metal ions, lipid peroxidation, gene expression, and DNA fragmentation simultaneously from genetically engineered mice brain regions and cultured neurons [36]. This study was conducted to improve conventional time-consuming, cumbersome, and less efficient procedures, and reduce 
the cost of conducting kinetic studies in the rare biological samples. The improved method was novel, precise, efficient, accurate, sensitive, economical, versatile, and highly reproducible. The recovery and shelf life of $\mathrm{CoQ}$ homologues was significantly improved and the chromatograms exhibited reduced $\mathrm{BKg}$ and retention times. In addition to $\mathrm{CoQ}$ homologues, this method could be utilized for DNA, RNA and proteins analyses from genetically rare, clinically significant biopsy, and autopsy samples.

Cross-breeding of $\mathrm{wv} / \mathrm{wv}$ female mice with $\mathrm{MT}_{\text {trans }}$ male mice provided a colony of MTsover-expressing wv/wv (wv/wv-MTs) mice, which had significantly high striatal CoQ 10 . These findings confirmed that MTs provide $\mathrm{CoQ}_{10}$-mediated neuroprotection by inhibiting free radicalinduced CB formation. Thus MTs gene-manipulated experimental mice and cultured human dopaminergic (SK-N-SH, SH-S-Y5Y) cells proved promising tools to evaluate the molecular pathogenesis of $\mathrm{CB}$ in health and disease.

Furthermore, we raised control-homozygous weaver mutant (wv/wv), and heterozygous weaver mutant (wv/+) mice to explore the basic molecular mechanism of neurodegeneration and the neuroprotective role of $\mathrm{CoQ}_{10}[38]$. wv/wv mice exhibited progressive neurodegeneration in the hippocampus, striatum, and cerebellum, and a reduction in the striatal dopamine and CoQs $\left(\mathrm{Q}_{9}\right.$ and $\left.\mathrm{Q}_{10}\right)$ without any significant changes in NE and 5-HT. Mitochondrial complex-1 was

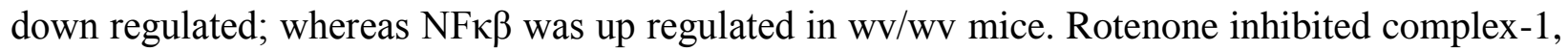

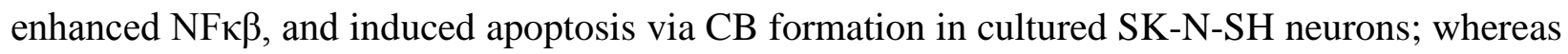

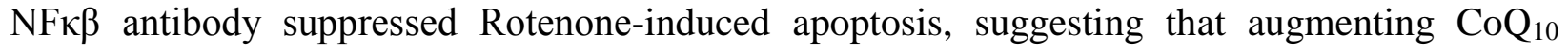
synthesis and inhibiting $N F \kappa \beta$ induction may provide neuroprotection in PD, AD, and aging.

\section{Main Hypotheses of $A D$}

Two main hypotheses have been proposed in the etiopathogenesis of AD. These are (a) Amyloid- $\beta$ (A $\beta-1-42)$ Hypothesis and (b) Mitochondrial hypothesis. According to amyloid hypothesis, $\mathrm{AD}$ occurs due to abnormal accumulation of $\mathrm{A} \beta-1-42$ in the senile plaques in the cortical ribbon. This hypothesis has been confirmed by detecting A $\beta-1-42$ senile plaques in the autopsy samples of AD patients using Congo-Red and by immunohistochemical analyses using specific $A \beta-1-42$ antibody in the senile plaques. Additionally, senile plaques can be detected in vivo by ${ }^{18} \mathrm{~F}$-PiB $\left({ }^{18} \mathrm{~F}\right.$-Florbetapir) PET neuroimaging in $\mathrm{AD}$ patients. The $\mathrm{A} \beta-1-42$ hypothesis was further confirmed by observing progression of neurobehavioral symptoms with neurodegeneration and cognitive impairment corresponding to the number of amyloid- $\beta$ 1-42 containing senile plaques. Nevertheless, several AD patients do not possess amyloid- $\beta$ plaques in their brain, yet exhibit progressive cholinergic deficit as a function of time, indicating the involvement of some other unknown molecular mechanism of neurodegeneration in AD.

We proposed MAOIs-mediated neuroprotection through $\mathrm{CB}$ inhibition which has been described in greater detail in one of the author's book [19]. There is currently considerable evidence in support of this hypothesis. The brain regional mitochondrial bioenergetics can be evaluated by performing ${ }^{18} \mathrm{FdG}$ PET neuroimaging. AD patients exhibit distinct loss of glucose metabolism in the fronto-temporal regions, ventriculomegaly, and hippocampal atrophy along with callosal and cerebral atrophy. Early manifestation of CB formation can be evaluated by estimating fluid biomarkers such as 8-OH, 2DG, lactate, glutamate, Choline, and N-Acetyl Aspartate (NAA) as rudiments of CB formation. Additionally, platelets, lymphocytes, buccal 
cells, and skin cells can be cultured and used to examine $\Delta \Psi$ collapse by using JC-1 or Rhodamine as sensitive fluorescent indicator to assess the mitochondrial bioenergetics and CB formation/inhibition in $\mathrm{AD}$ patients. In general, the mitochondrial hypothesis relies on the structural and functional integrity of the mitochondrial bioenergetics which may be compromised due to oxidative and nitrative stress. Oxidative and nitrative stress occur due to free radical overproduction in the mitochondria as a byproduct of oxidative phosphorylation during ATP synthesis in the electron transport chain. Free radicals-induced lipid peroxidation causes structural and functional breakdown of polyunsaturated fatty acids (Linoic acid, Linolinic acid, and Arachidonic acid) in the mitochondrial plasma membranes to induce CB formation in AD. Although the free radical theory of mitochondrial neurodegeneration is well established, we discovered for the first time the basic molecular mechanism of MAOIs-induced neuroprotection through MTs-mediated CB inhibition. Accordingly, this manuscript is unique and has distinct merits compared to already existing publications because of the following considerations:

(a) Mitochondrial oxidative phosphorylation in the electron transport chain is involved in ATP synthesis.

(b)Free radicals $(* \mathrm{OH}, \mathrm{NO})$ are generated as a byproduct of oxidative phosphorylation in the mitochondria.

(c) ATP is required for the synthesis of S-adenosyl methionine by S-adenosyl-methionine transferase.

(d)S-adenosyl methionine is involved in the DNA methylation at the cytosine residue and histone acetylation at the lysine residue. These epigenetic changes are involved in numerous known and unknown aspects of health and disease including; neurodegenerative diseases, cardiovascular diseases, and cancer.

(e) The distinct advantage of antioxidants derived from functional foods is that they can pass blood brain barrier (BBB) without causing adverse side effects, unlike currentlyavailable antidepressants, antipsychotic, anti-histaminergic, cholinesterase and NMDA receptor antagonists used in the treatment of $\mathrm{AD}$.

(f) Although CB formation occurs based on the principle: "Similar molecules associate, whereas dissimilar molecules dissociate"; this molecular association remains compromised and $\mathrm{CB}$ is unable to function as healthy mitochondria. Therefore, early prevention and/or inhibition of $\mathrm{CB}$ formation by life-style modification may be considered as a crucial step in healthy aging and novel drugs development to enhance charnolophagy as a basic molecular mechanism of intracellular detoxification.

(g)Free radicals are generated as a byproduct of oxidative phosphorylation in the mitochondria. Nutritional stress, drug addiction, and/or environmental toxins (heavy metals, microbes) enhance free radical overproduction due to increased mitochondrial energy demands and oxidative/nitrative stress.

(h)Free radical overproduction enhances lipid peroxidation to induce structural and functional breakdown of polyunsaturated fatty acids in the plasma membranes to cause mitochondrial degeneration.

(i) The degenerated mitochondrial membranes condense to form pleomorphic $\mathrm{CBs}$, implicated in impaired axoplasmic transport of various enzymes, hormones, 
neurotransmitters, neurotropic factors (BDNF, NGF, IGF-1) and mitochondria, and hence abnormal neurotransmission at the synaptic terminals to cause cognitive impairments in $\mathrm{AD}$ and other neurodegenerative diseases.

(j) Antioxidants such as MTs and functional foods are able to inhibit mtDNA oxidation, 8$\mathrm{OH}, 2 \mathrm{dG}$ synthesis, and epigenetic changes to prevent $\mathrm{CB}$ formation involved in progressive neurodegeneration. The mtDNA is a single stranded, intron-less, and devoid of histones and protamines, and remains in a hostile environment of free radicals; thus, is highly susceptible to oxidative and nitrative stress; is likely to be destroyed more readily as compared to nuclear DNA, which is double stranded, helical structure with remarkable protection from the double layered nuclear wall, nucleoplasm, histones, and protamines. Additionally, the nuclear DNA has noncoding introns, which minimize the chances of uncoiling, direct exposure to free radicals, and undesirable hypermethylation of gene promoter regions and global hypomethylation, involved in progressive neurodegenerative disorders, cardiovascular disorders, and cancer.

(k)The down-regulation of mitochondrial as well as nuclear genome during oxidative and nitrative stress in highly vulnerable cells such as hippocampal CA-3 and dentate gyrus neurons is involved in impaired NMDA and glucocorticoid receptor-mediated long term potentiation (LTP) and memory consolidation in AD. Therefore, novel drugs may be developed to boost the mitochondrial bioenergetics and prevent/inhibit CB formation.

(1) Since neuronal replacement therapy and/or radical surgery of CNS is not currently a better option, neuro-restorative therapy with either endogenous antioxidants such as MTs, glutathione, SOD, and catalase) or exogenous antioxidant derived from functional foods containing Flavonoids, Polyphenols, Sirtuins, Rituins, Reseveratrol, Catchsin, and other inhibitors of lysine deacetylases (LSDs), and $\mathrm{CoQ}_{10}$, may have significant therapeutic potential to preserve the mitochondrial bioenergetics, inhibit $\mathrm{CB}$ formation, and hence progressive neurodegeneration in $\mathrm{PD}, \mathrm{AD}, \mathrm{MDD}$, and drug addiction.

(m) The distinct advantage of functional foods is that they are rich in antioxidants and can easily pass through blood brain barrier, without causing any deleterious central or peripheral adverse effects in the aging brain, as there is currently limited promise of neuron replacement therapy and/or radical neurosurgery due to slow neuro-recovery and regenerative potential.

\section{Pyridoxine Attenuates Domoic Acid-Induced Hippocampal CB Formation}

We reported that $\mathrm{CB}$ formation occurs in the most vulnerable cells (like cerebellar Purkinje neurons and hippocampal CA-3 and dentate gyrus neurons) due to nutritional and/or environmental (toxins) stress in the developing or aging brain $[10,11,26]$. A specific $5-5 \mathrm{~T}_{1 \mathrm{~A}}$ agonist, 8-OH-DPAT suppressed Domoic acid-induced seizure activity and progressive neurodegeneration, whereas $5 \mathrm{HT}_{1 \mathrm{~A}}$ receptor antagonist, Spiroxatrine augmented seizure discharge activity and augmented hippocampal CB formation. Thus, CB as an early and sensitive pre-apoptotic biomarker of compromised bio-energetics supports the mitochondrial hypothesis of $\mathrm{AD}$ progression, and further suggests the neuroprotective and therapeutic potential of exogenous and/or endogenous antioxidants in AD, such as MTs, as we have established. 


\section{Vitamin $B_{6}$ (Pyridoxine)-Mediated CB Inhibition Confers Neuroprotection}

We reported that cerebral cortex of mice administered a single sub-convulsive dose of Domoic acid induces electrical seizure discharge, associated with typical spike and wave activity, increases in $\Delta$ and $\Theta$ and reductions in $\alpha$ and $\beta$, as determined by frequency and power spectral analyses of computerized EEG [39]. Administration of Sodium Valproate, Nimodipine, or $5 \alpha$ Pregnan $3 \alpha$-ol-20-one as well as Pyridoxine suppressed Domoic acid-induced spike and wave activity and ameliorated abnormal EEG frequencies. Administration of these drugs 45 min prior to the administration of Domoic acid also significantly ameliorated abnormal EEG. Sodium valproate and pyridoxine significantly attenuated Domoic acid-induced increase in glutamate and calcium influx, decrease in GABA and increase in the protooncogenes c-fos, jun-B and jun-D expression. Domoic acid-induced increases in glutamate and calcium influx in the hippocampal neurons were decreased by vitamin $\mathrm{B}_{6}$ or Nimodipine. Similarly, in cultured NG 108/15 cells, vitamin $\mathrm{B}_{6}$ suppressed Domoic acid-induced increases in glutamate, $\left[\mathrm{Ca}^{2+}\right]_{\mathrm{i}}$, and oncoproteins expression regardless of whether cells were undifferentiated, differentiated or dedifferentiated. Vitamin $\mathrm{B}_{6}$ had anti-seizure and neuroprotective actions. Vitamin $\mathrm{B}_{6}$ (Pyridoxine) is converted to pyridoxal by an enzyme, pyridoxine oxidase and pyridoxal is converted to pyridoxal phosphate (PLP) by enzyme, pyridoxal kinase. PLP participates in numerous decarboxylation reactions as a coenzyme for the synthesis of neurotransmitters such as GABA, dopamine, 5-HT, and Taurine, involved in cognitive performance. Later on we discovered that in the vitamin $\mathrm{B}_{6}$ deficiency-induced hypertensive (B6DHT) rat there is an increased influx of calcium into the vascular smooth muscle cells. Vitamers, which possess vitamin $\mathrm{B}_{6}$ activity, blocked calcium influx into the caudal artery segments of B6DHT rats and the BAY K 8644induced influx into artery segments from vitamin $\mathrm{B}_{6}$-supplemented (control) rats. No further increase was noticed in the calcium influx from B6DHT rats in response to BAY K 8644. However, when this influx was decreased by Nifedipine or by PLP, BAY K 8644 antagonized their effects, indicating that calcium influx mediated by the dihydropyridine-sensitive calcium channel is a site of the calcium influx defect in the B6DHT rat [40]. It is now established that PLP plays a crucial role in the nervous system as the amino acid decarboxylases involved in the synthesis of all electro-physiologically-significant neurotransmitters require PLP as a coenzyme. Vitamin $\mathrm{B}_{6}$ and its active vitamers possess antioxidant properties. PLP has a role in regulating cellular calcium transport through both the voltage-operated and ATP-mediated purinergic mechanisms of cellular calcium influx and, hence, has a role controlling hypertension. Pharmacological doses of vitamin $\mathrm{B}_{6}$ decreased the high blood pressure associated with both genetic and acquired models of hypertension, suggesting that Pyridoxine has a crucial role in the normal function of the central and peripheral nervous systems. It also protects against ischemia and glutamate-induced neurotoxicity [41].

\section{Selegiline-Mediated CB Inhibition Confers Neuroprotection}

It is now well-established that Selegiline, a selective inhibitor of MAO-B, is one of the first adjunct therapies in clinical neurology. A retrospective analysis of data from patients with PD exhibited a significant increase in survival in those treated with Selegiline plus L-DOPA compared with L-DOPA alone. We highlighted that the basic molecular mechanism of action of Selegiline is complex and cannot be explained exclusively based on its MAO-B inhibitory 
action because pretreatment with Selegiline can protect neurons against a variety of neurotoxins, such as 1-methyl-4-phenyl-1,2,3,6 tetrahydropyridine (MPTP), 6-hydroxy-dopamine, N-(2chloroethyl)-N-ethyl-2-bromobenzylamine (DSP-4), methyl-beta-acetoxyethyl-2chloroethylamine (AF64A), and 5,6-dihydroxyserotonin, which destroy dopaminergic, adrenergic, cholinergic, and serotonergic neurons, respectively. Selegiline produces an amphetamine-like effect, enhances dopamine release, and blocks the reuptake of dopamine. It stimulates gene expression of L-aromatic amino acid decarboxylase, increases striatal phenylethylamine levels, and activates dopaminergic receptors. Selegiline reduces oxidative radicals, up-regulates SOD and catalase, and suppresses nonenzymatic and iron-catalyzed oxidation of dopamine. Selegiline compensates for loss of target-derived trophic support, delays apoptosis in serum-deprived cells, and blocks apoptosis-related $\Delta \Psi$ collapse and $\mathrm{CB}$ formation $[42,43]$.

We examined mitochondrial membranes and molecular biomarkers of apoptosis in response to increasing concentrations of 1-methyl, 4-phenyl, pyridinium ion ( $\mathrm{MPP}^{+}$) in $\mathrm{SK}-\mathrm{N}-\mathrm{SH}$ neurons and evaluated the neuroprotective potential of Selegiline to explore its molecular mechanism(s) of neuroprotection [20]. $\mathrm{MPP}^{+}$-induced apoptosis was characterized by spherical appearance, suppressed neuritogenesis, phosphatidyl serine externalization, plasma membrane perforations, $\Delta \Psi$ collapse, CB formation, and DNA fragmentation and condensation. At lower concentrations, $\mathrm{MPP}^{+}(10-100 \mu \mathrm{M})$ produced mitochondrial swelling and loss of cristae, and at higher concentrations (300-500 $\mu \mathrm{M})$, degenerated and aggregated the membranes in the peri-nuclear region as pleomorphic $\mathrm{CBs}$, which were attenuated by Selegiline (10-50 $\mu \mathrm{M})$ pre-treatment. At still higher concentrations, $\mathrm{MPP}^{+}(>500 \mu \mathrm{M})$ produced necrotic changes represented by mitochondrial and plasma membrane ballooning and perforations. Selegiline provided partial neuroprotection at higher concentrations of $\mathrm{MPP}^{+}$. $\mathrm{MPP}^{+}$-induced increases in ROS, lipid peroxidation, cytochrome-C release, NF- $\kappa \beta$ activation, $8-\mathrm{OH}, 2 \mathrm{dG}$ synthesis, $\alpha$-synuclein indices, and reductions in glutathione, ATP, and SOD were attenuated by Selegiline. Selegiline also attenuated $\mathrm{MPP}^{+}$-induced transcriptional activation of c-fos, c-jun, GAPDH, and caspase-3, suggesting that it may provide neuroprotection by preserving mitochondrial membranes, by attenuating molecular biomarkers of apoptosis, by scavenging free radicals, and by regulating immediate early genes involved in neurodegeneration. In a subsequent study, we established that Selegiline enhances MTs in the CNS, which prevent CB formation by acting as free radical scavengers. In order to further confirm free radical theory of progressive neurodegeneration, we examined the direct effect of a ONOO- ion generator, 3-morpholino-sydnonimine (SIN-1) in cultured human dopaminergic (SK-N-SH) neurons. SIN-1 induced apoptosis by causing mitochondrial dysfunction, whereas Selegiline prevented mitochondrial dysfunction by preventing free-radical-induced CB formation [44, 45].

\section{Free Radical Theory of Progressive Neurodegeneration}

The iron released from the degenerated Cyt-C participates in the Fenton reaction to form potent $\mathrm{ONOO}^{-}$ions from $* \mathrm{OH}$ and $* \mathrm{NO}$ radicals, which induces oxidative in addition to nitrative stress, causing further neurodegeneration. MAO-B inhibitors, Selegiline and Rasagiline provide neuroprotection by enhancing MTs expression, which as free radical scavengers, attenuate $\mathrm{ONOO}^{-}$-induced oxidative and nitrative stress to inhibit $\mathrm{CB}$ formation, implicated in early phase 
of neurodegeneration. Based on two types of MAOs, we proposed two types of CBs in the CNS including: MAO-A-specific CBs, and MAO-B-specific CB. Thus, novel therapeutic agents to target MAO-A-specific $\mathrm{CB}$ will have great promise in the effective treatment of MDDs and anxiety disorders whereas $M A O-B$-specific $C B$ targeting agents will be beneficial for the clinical management of PD and AD. These drugs will have superior PKs, PDs, and promising therapeutic potential because of increased sensitivity, specificity, and targetability with minimum or no adverse effects as noticed in currently available MAOIs, anti-carcinogenic drugs, and cardiovascular drugs. Various antidepressants including MAOIs and antipsychotic drugs have reduced therapeutic index and reduced margin of safety. In general, the MAOIs used in clinical practice have serious adverse effects including: hypertensive crisis and/or 5-HT toxicity particularly when consumed along with Tyramine-rich foods and specific serotonin reuptake inhibitors (SSRIs) such as Fluoxetine and Paroxetine. Although it is claimed that the new generation of selective MAOIs are quite safe with superior pharmacological profile, it is exceedingly important to emphasize that, these drugs must be prescribed by an experienced physician with sufficient basic knowledge to avoid any fatal incidence of hypertensive crisis, 5HT toxicity, suicide ideation, and/or other undesirable self-mutilation tendencies.

CB formation in different cells (including skin cells, buccal mucosa, vaginal smear, neurons, lymphocytes, and platelets) can be studied to explore the peripheral diagnosis of AD. More specifically, the author discussed in-vitro biomarkers of body fluids including; CSF, serum, and blood platelets. We established that brain regional augmentation of $\mathrm{CoQ}_{10}$ and/or MTs provide neuroprotection by inhibiting free radical-induced $\mathrm{CB}$ formation in the most vulnerable nigrostriatal DA-ergic neurons in $\mathrm{PD}$ and hippocampal cholinergic neurons in AD. Hence, microPET neuroimaging with ${ }^{18} \mathrm{~F}$-DOPA and ${ }^{18} \mathrm{FdG}$ in MTs gene-manipulated mice may provide better insight in understanding the exact cellular, molecular, and genetic basis of CB-mediated progressive neurodegenerative diseases, and their personalized theranostics with minimum or no adverse effects.

\section{Assessment of Pre-apoptosis by In-Vivo Imaging of MAO-Specific CB Formation}

Recently we proposed two types of CBs in the CNS and other tissues depending on the type of MAOs localized on the outer mitochondrial membranes. MAO-A specific CB formation can occur primarily in the NE-ergic and 5-HT-ergic neurons in the dorsal raphe and periaqueductal regions in the CNS, which is involved in pain perception and mood fluctuations, whereas MAO$\mathrm{B}$-specific $\mathrm{CB}$ is formed in the NS-DA-ergic neurons, which possess MAO-B on the outer mitochondrial membranes. Thus, pre-apoptotic events at the mitochondrial levels can be detected by performing non-invasive, in-vivo imaging with ${ }^{11} \mathrm{C},{ }^{18} \mathrm{~F}$, or ${ }^{124} \mathrm{I}$ radiolabeled Selegiline and Rasagiline or by any other selective MAO inhibitor to assess early pre-apoptotic events during $\mathrm{PD}$, AD, drug addiction, stroke, TBI, PTSD, ADHD, and other neurodegenerative diseases, cardiovascular diseases, and cancer. A further research in this direction will facilitate early detection of these disorders to accomplish long-quested evidence-based personalized theranostics (EBPT) of these devastating illnesses [46, 47].

\section{CB as a Novel Biomarker in Zika Virus Induced Microcephaly}


The brain develops almost 20 times faster compared to other organs during first trimester of pregnancy; hence it is highly vulnerable to environmental or toxic insults, including Zika virus infection. Zika virus is a mosquito-borne infection related to dengue, yellow fever, and West Nile virus. Currently, it remains uncertain how Zika Virus infection causes microcephaly and there is no vaccine available against its infection. Zika Virus was first detected in Pernambuco (Brazil) in May 2015. About 62 locations mostly in the Caribbean, Central America and South America have been affected by Zika Virus infection. The children exposed to Zika Virus infection may become victims of microcephaly and burden to society rest of their life. The author proposed that Zika Virus infection in utero compromises mitochondrial bioenergetics to enhance free radical overproduction and lipid peroxidation in the highly vulnerable developing neurons to enhance CB formation and apoptosis; which may eventually induce microcephaly. Thus, early therapeutic interventions to inhibit CB formation by nutritional rehabilitation, antioxidants, and healthy life-style choices (including personal and environmental hygiene) and refraining from drugs of abuse including smoking and alcohol may go a long way in the prevention and/or treatment of Zika Virus-induced or other forms of microcephaly in utero. Various risk factors including (i) fetal alcohol, environmental neurotoxins (poly-chlorobiphenyls: PCBs, Lead: Pb, and Mercury, $\mathrm{Hg}$ ), and Zika viral infection alone or in combination may induce CB formation in the developing neurons to cause apoptosis, and eventually microcephaly. Thus, environmental protection, personal hygiene, well-nourished diet, and antioxidants may attenuate CB formation to prevent microcephaly. The author discovered CB initially in the developing undernourished rat cerebellar Purkinje neurons and in the intrauterine Domoic acid-exposed mice hippocampal and hypothalamic neurons. The incidence of $\mathrm{CB}$ is increased with the severity of nutritional and environmental neurotoxic insult. We reported $\mathrm{CB}$ as a universal biomarker of cell injury in nanomedicine and chronic drug addiction. Subsequently, CB was detected in various cellular and animal models of FAS, PD, AD, vascular dementia, chronic drug addiction, and during intrauterine exposure to environmental neurotoxins such as Kainic Acid, Domoic acid, Acromelic Acid, PCBs, Lead, Arsenic, and Mercury. At the ultrastructural level, CB appears as a pleomorphic, electron-dense multi-lamellar, quasi-crystalline, stack of degenerated mitochondrial membranes causing progressive neurodegeneration in highly vulnerable neurons of the developing brain and may be induced by Zika Virus infection during intrauterine life from infected parents. CB is a pre-apoptotic biomarker of compromised mitochondrial bioenergetics and is formed in response to intrauterine infection, nutritional stress, environmental toxins, and/or drugs of abuse due to free radical overproduction and mitochondrial genome downregulation. Accumulation of $\mathrm{CB}$ at the junction of axon hillock impairs axoplasmic flow of various enzymes, neurotransmitters, hormones, neurotropic factors (NGF, BDNF), and mitochondria at the synaptic terminals to cause cognitive impairment, early morbidity, and mortality accompanied with or without microcephaly. Early events in CB formation including: $\Delta \Psi$ collapse, down-regulation of mitochondrial ubiquinone-NADH-oxidoreductase (complex-1) and $8-\mathrm{OH}-2 \mathrm{dG}$ can be detected in the blood, saliva, semen, and urine samples as rudiments of CB to evaluate epigenetic modifications of DNA methylation and histone acetylation following cellular injury with or without microbial infection. During chronic phase, CB can be detected at the ultrastructure level in the platelets, lymphocytes, neurons, oocyte, spermatocyte, and in any highly vulnerable cell from the infected parents as well as from the newborn child. Antioxidants 
such as MTs inhibit CB formation as free radical scavengers by regulating zinc-mediated transcriptional activation of genes involved in growth, proliferation, and differentiation, as we discovered in gene-manipulated human dopaminergic (SK-N-SH and SHY5Y) cells and in mouse models of neurodegeneration and multiple drug abuse [48].

Consequently, novel drugs may be developed to prevent $\mathrm{CB}$ formation or enhance charnolophagy as the basic molecular mechanism of intracellular detoxification during acute phase and CB antagonists to avert microcephaly, FAS, or autism by employing CB as an early, sensitive and specific biomarker to detect, prevent and effectively treat microcephaly in early days and in aging. Based on two types of monoamine oxidases on the outer mitochondrial membranes, two types of $\mathrm{CBs}$ were proposed (i.e., MOA-specific $\mathrm{CB}$, and MOA-B-specific $\mathrm{CB}$ ), which can be targeted in vivo by ${ }^{11} \mathrm{C}$ or ${ }^{18} \mathrm{~F}$-labeled monoamine oxidase-A or $\mathrm{B}$ inhibitor (Selegiline) to detect $\mathrm{CB}$ formation in the developing brain, in addition to ${ }^{18} \mathrm{FdG}-\mathrm{PET}$ neuroimaging for in-vivo assessment of brain regional mitochondrial bioenergetics. However, radiation exposure to developing embryo will be a major concern which needs to be resolved to accomplish EBPT of Zika virus-induced or other forms of microcephaly in the developing brain. $\mathrm{CB}$ was discovered as an early, pre-apoptotic biomarker of compromised mitochondrial bioenergetics in progressive neurodegenerative disorders and can be detected at an earlier stage of neuronal impairment. Currently novel biomarkers are being developed to define the preclinical stages of AD. It is now emphasized that the primary objective of CNS drug development is to elucidate the molecular mechanism rather than molecule to extend further research on clinical trials. PET imaging biomarkers provide confirmation of targeted drug delivery for neuropsychiatric drug development and facilitate exploring the neuropharmacological basis of psychiatric diseases and the optimization of drug therapy.

\section{MicroRNAs as CB Modulators}

Recently various types of microRNAs were proposed to serve neurodegenerative and neuroprotective roles. However, their exact significance in CB induction or inhibition in health and disease is yet to be established.

\section{MicroRNA, CB, and AD}

The discovery of miRNAs evoked excitement in biomedical research due to their promise as potential disease biomarkers and therapeutic targets. miRNAs are now recognized as key players in virtually every biological process. miRNAs are single-stranded, small non-coding RNA regulators of protein synthesis that function as "fine-tuning" tools of gene expression in development and tissue homeostasis. microRNAs are involved in post-transcriptional control of a wide variety of genes. miRNAs align and bind especially to 3'UTR sequences of their target genes and initiate either mRNA degradation or translational repression, resulting in reduced protein synthesis. Impaired microRNA-mediated regulation is implicated in the pathogenesis of $\mathrm{AD}$ and cancer [49]. miRNAs are post-transcriptional gene expression regulators and key players in the neuronal development, plasticity, and disease and are one of the major noncoding RNAs that function as negative regulators to silence or suppress gene expression via translational inhibition or message degradation. Their profiles are significantly altered in neurodegenerative diseases such as $\mathrm{AD}$ that is characterized by both amyloid- $\beta(\mathrm{A} \beta)$ and tau deposition in brain 
[50]. How changes in miRNA profiles translate into biological function in a physiological and pathological context are yet to be established. The key lies in identifying specific target genes for deregulated miRNAs and understanding which pathogenic factors trigger their deregulation. The researchers reviewed the literature about the intricate network of miRNAs surrounding the regulation of the amyloid precursor protein (APP) from which $\mathrm{A} \beta$ is derived by proteolytic cleavage. Normal brain function is highly sensitive to any changes in APP metabolism and miRNAs function to ensure the correct APP end product is produced and in the right form and abundance. Disruptions in the miRNA regulatory network may alter A $\beta$ production, which in turn can affect miRNA expression. MicroRNAs are essential for various cellular events including cell lineage determination, proliferation, apoptosis, DNA repair, and cytoskeletal organization to fine-tune gene expression at the post-transcriptional level in various cell signaling mechanisms. Recently it was reported that miRNAs are present in mitochondria, regulate their functional activity, and are deregulated in various diseases including AD and cancers [51]. Reprogramming of the metabolism is postulated as a major feature of cancer. miRNAs modulate various metabolic pathways involved in tumor suppression. As a result, modulation of miRNAs provides a novel therapeutic approach for AD and cancer treatment.

\section{Role of microRNAs in Mitochondrial Function and AD}

Recently Duarte et al [52] reported that the discovery of miRNAs has revolutionized the conventional view of gene expression and our understanding of miRNA biogenesis and function has thereby expanded. The mitochondrial-located miRNAs raised the issue of the molecular mechanism underlying their translocation from the nucleus to the mitochondria. It may exist a number of pathways of nucleus-encoded RNAs to mitochondria, being the ATP-dependent. Not only pre-miRNAs, but also mature miRNAs, are present in the mitochondria; these findings raised the possibility of mitochondrial miRNA synthesis. Some pre-miRNAs sequences are processed in the mitochondria, giving origin to mature miRNAs, which could be active on the mitochondrial transcripts or exported to the cytosol to interfere with genomic-derived mRNA. Thus, the mitochondrial-processed miRNAs may contribute to post-transcriptional regulation of genes involved in mitochondrial functions., Some mitochondrial miRNAs are currently named as mitomiRs; as these are localized in mitochondria, whether transcribed by the nuclear and/or, the mitochondrial genome. A number of mitomiRs mapped the nuclear genome at loci relevant to mitochondrial functions or diseases. Computational analyses, using different algorithms demonstrated that the mitochondrial genome can harbor sequences that could be a target for several mitomiRs. However, a more challenging topic concerning mitomiRs is whether the mtDNA can harbor miRNA sequences, to suggest an involvement of mitochondria in small RNA-generating pathways. The detection of miRNAs in the mitochondria has encouraged to explore its biological functions [52]. miRNAs, originated in the nuclear genome, are exported to cytosol where they are processed and exert their function by inhibiting nuclear genome-derived mRNA. Some miRNAs are imported into mitochondria where they interact with some mitochondrial genome-derived mRNA molecules. Mitochondrial genome (mtDNA) can originate some miRNA molecules that exert their function directly on mitochondrial transcripts. The links between miRNA deregulation and human disease have been reported in various biomedical fields. Currently, efforts are being made in understanding the involvement of miRNA 
deregulation in disease and discovering molecular mechanisms by which they execute their functional activity. This emerging field has revealed the potential of miRNAs as diagnostic or even as valuable therapeutic tools. miRNAs have recently emerged as key regulators of metabolism. Metabolic syndrome is a systemic disorder that includes abnormalities associated with obesity and type II diabetes. Defects in mitochondrial function, related to oxidation of fatty acids, have been linked to diet-induced obesity and the development of insulin resistance in adipose tissue and skeletal muscle. Consistently, obese subjects have mitochondria with compromised bioenergetics. Therefore, increasing interest is being given to the role of miRNAs on metabolic regulation, with relevance on mitochondria and the mechanisms for miRNA actions, particularly acting in mitochondria or in mitochondria-related pathways. The involvement of miRNAs in mitochondrial metabolism, oxidative phosphorylation (OXPHOS), electron transport chain (ETC) components, lipid metabolism, and metabolic disorders is becoming more comprehensive, as well as miRNAs contribution for mitochondrial dynamics or apoptosis regulation and cancer. However, the exact pathophysiological significance of miRNAs in CB induction/repression remains enigmatic. A blood based 12-miRNA signature of Alzheimer disease patients has been recently discovered [53].

\section{Pro-inflammatory, Anti-inflammatory, and Anti-Apoptotic Roles of microRNA in AD}

Recently pro-inflammatory, anti-apoptotic, and anti-inflammatory miRNA was elucidated [54]. It was reported that neuro-inflammation, one of the pathogenic causes of neurodegenerative diseases, is regulated through the cholinergic anti-inflammatory pathway via the $\alpha 7$ nicotinic acetylcholine receptor $(\alpha 7 \mathrm{nAChR})$. These investigators demonstrated that either bacterial lipopolysaccharide (LPS) or immunization with the $\alpha 7(1-208) n A C h R$ fragment decrease $\alpha 7$ nAChRs density in the mouse brain, exacerbating chronic inflammation, $A \beta$ accumulation and memory decline, which mimic the early stages of AD. To study the molecular mechanisms underlying the LPS and antibody effects in the brain, these investigators employed an in vivo model of LPS-induced inflammation and an in vitro model of cultured glioblastoma U373 cells. LPS challenge decreased the levels of $\alpha 7 \mathrm{nAChR}$ RNA and protein and of acetylcholinesterase (AChE) RNA and activity in distinct mouse brain regions, sensitized mitochondria to the apoptogenic effect of $\mathrm{Ca}^{2+}$ and modified brain microRNA profiles, including the cholinergicregulatory CholinomiRs-132/212, in favor of anti-inflammatory and pro-apoptotic ones. Adding $\alpha 7(1-208)$-specific antibodies to the LPS challenge prevented elevation of anti-inflammatory and pro-apoptotic miRNAs while supporting the resistance of brain mitochondria to $\mathrm{Ca}^{2+}$ and maintaining $\alpha 7 \mathrm{nAChR} / \mathrm{AChE}$ decreased. In U373 cells, $\alpha 7$-specific antibodies and LPS induced IL-6 production through the p38/Src-dependent pathway, suggesting that acute LPS-induced inflammation induces the cholinergic anti-inflammatory pathway, that $\alpha 7 \mathrm{nAChR}$ downregulation limits this pathway, and that $\alpha 7$-specific antibodies aggravate neuro-inflammation by inducing the pro-inflammatory IL-6 and dampening anti-inflammatory miRNAs; however, these antibodies may protect brain mitochondria and decrease pro-apoptotic miRNAs, preventing LPSinduced neurodegeneration through CB formation.

Mitochondrial Malate Dehydrogenase Upregulation by Oxidative Stress is Mediated by miR$743 a$. 
Shi and Gibson [55] discovered that miRNAs mediate oxidant regulated expression of a mitochondrial tricarboxylic acid cycle gene $(\mathrm{mdh} 2)$. mdh2 encoded malate dehydrogenase $(\mathrm{MDH})$ was elevated in brains of patients that died with $\mathrm{AD}$. Oxidative stress, an early and pervasive event in $\mathrm{AD}$, increased $\mathrm{MDH}$ activity and mRNA of mdh2 by $19 \%$ and $22 \%$, respectively, in a mouse hippocampal cell line (HT22). Post-transcriptional events underlie the change in mRNA because Actinomycin D did not block the elevated mdh2 mRNA. Since miRNAs regulate gene expression post-transcriptionally, the expression of miR-743a, a miRNA to target mdh2, was determined and showed a 52\% reduction after oxidant treatment. Direct interaction of miR-743a with mdh2 was demonstrated with a luciferase based assay. Overexpression or inhibition of miR-743a led to a reduction or increase in endogenous mRNA and MDH activity, suggesting that miR-743a negatively regulates $\mathrm{mdh} 2$ at post-transcriptional level by directly targeting the mdh2 3'UTR and that oxidative stress can increase the activity of MDH through miR-743a, and provide new insights into possible roles of miRNA in oxidative stress and neurodegeneration.

\section{ER Stress in $A D$}

It has been reported that ER stress is implicated in the pathophysiology of many diseases including heart disease, cancer, and neurodegenerative diseases such as AD and HD [56]. Prolonged or excessive ER stress results in the induction of signaling pathways resulting in cell death. There is now better understanding of the signaling pathways leading to ER stressmediated cell death and the importance of ER localized stress sensors, IRE1 $\alpha$, ATF6 and PERK in this process. These investigators provided current concepts of various stages of ER stressinduced cell death, focusing on the role of ER localized proteins in sensing and triggering ER stress-induced death signals with emphasis on the role of calcium signaling and Bcl-2 family members. They also highlighted ER stress-induced cell death, particularly the significance of microRNAs, ER-mitochondria cross talk, and the mitochondria-independent death signals in ER stress-induced cell death.

\section{DRP Fission Proteins in Mitochondria in Relation to AD}

Although mitochondrial abnormalities have been documented in $\mathrm{AD}$ and related neurodegenerative disorders, the causal relationship between mitochondrial changes and neurodegeneration, and the specific mechanisms promoting mitochondrial dysfunction, remain uncertain. It has been discovered that the human tau expression causes elongation of mitochondria in both Drosophila and mouse neurons [57]. Elongation was accompanied by mitochondrial dysfunction and apoptosis, which could be rescued in vivo by genetically restoring the mitochondrial fission and fusion. These researchers demonstrated that stabilization of actin by tau is critical for neurotoxicity of the protein and that actin and myosin are needed in regulating mitochondrial fission and that excess actin stabilization inhibits association of the fission protein DRP1 with mitochondria, leading to mitochondrial elongation and neurotoxicity. Their results thus identified actin-mediated disruption of mitochondrial bioenergetics as a direct molecular mechanism of tau toxicity in neurons in vivo.

\section{H1G1 as a Novel Regulator of Mitochondrial $\gamma$-Secretase}


The $\gamma$-secretase complex (which contains presenilins, nicastrin, anterior pharynx defective-1, and presenilin enhancer-2) cleaves type I transmembrane proteins, including Notch and amyloid precursor protein. Dysregulated $\gamma$-secretase activity has been implicated in the pathogenesis of $\mathrm{AD}$, stroke, atherosclerosis, and cancer. Hayashi et al [58] reported that regulation of $\gamma$ secretase activity is required for normal physiology. They isolated HIG1 (hypoxia inducible gene 1, domain member 1A) from $\gamma$-secretase inhibitory genes. HIG1 was highly expressed in the brain. Interestingly, HIG1 was localized in the mitochondria and was directly bound to $\gamma$ secretase on the mitochondrial membrane in SK-N-SH cells. Over-expression of HIG1 attenuated hypoxia-induced $\gamma$-secretase activation on the mitochondrial membrane and the accumulation of intracellular amyloid $\beta$. This accumulation was accompanied by hypoxiainduced mitochondrial dysfunction. The latter half domain of HIG1 was required for binding to the $\gamma$-secretase complex and suppression of $\gamma$-secretase activity. The depletion of HIG1 increased $\gamma$-secretase activation and enhanced hypoxia-induced mitochondrial dysfunction, suggesting that HIG1 is a novel modulator of the mitochondrial $\gamma$-secretase complex, and plays an important role in the maintenance of normal mitochondrial function.

\section{Role of Mitochondria in AD Pathogenesis}

Several studies have been conducted to establish the precise role mitochondria in maintaining neuronal function by providing energy, particularly at the synaptic terminals. Recently the role of Drp1 in AD pathogenesis and its relationships to glycogen synthase kinase 3, cyclin-dependent kinase 5, p53, and microRNAs in AD pathogenesis was highlighted [59]. It was suggested that $A \beta$ and phosphorylated tau interact with the mitochondrial fission protein, dynamin-related protein 1 (Drp1), causing excessive fragmentation of mitochondria, leading to abnormal mitochondrial dynamics and synaptic degeneration in $\mathrm{AD}$ neurons. Additionally, $\mathrm{A} \beta$-induced and phosphorylated tau induced changes in mitochondria, compromising their shape, size, distribution and axonal transport in $\mathrm{AD}$ neurons due to $\mathrm{CB}$ formation as we have highlighted in this report. These changes affect mitochondrial health, synaptic function, neuronal damage, and ultimately memory loss and cognitive impairment in $\mathrm{AD}$ patients.

\section{Abnormal Mitochondrial RNA in Aging and AD}

It is well established that accumulations of mtDNA mutations associated with aging are evident in multiple human tissues. The role of mtDNA mutations can be observed in an aging animal model such as homozygous knock-in PolgA mice, which have a colonial expansion of mtDNA mutations [60]. These mice have reduced lifespan and premature onset of age-related phenotypes, that are also observed in aging acceleration with anti-retroviral therapy through clonal expansion of mtDNA mutations. These clonally expanded mtDNA mutations maintain transcription ability which could result in an accumulation of abnormal mitochondrial RNA (mtRNA) in the affected cells. Compensation-effect doctrine states that mtDNA mutations in the cell must reach a set threshold before they have a negative impact on cellular function due to compensation from normal mtDNA. In contrast to this theory, these investigators suggested that an accumulation of aberrant mtRNA transcribed from mtDNA mutations negatively influences cellular function through complex mitochondrial pathways, and thereby may be an important cause of aging and aging-related diseases. 


\section{Glycosylation of Hemoglobin Facilitates CB Formation}

It is well established that glycosylation of hemoglobin impairs mitochondrial function by downregulating the $\Delta \Psi$ and mtDNA, and by increasing DNA oxidation to synthesize $8-\mathrm{OH}, 2 \mathrm{dG}$, which facilitates $\mathrm{CB}$ formation due to free radical-induced compromised mitochondrial bioenergetics. Glycosylation of hemoglobin also triggers epigenetic changes in the DNA to increase the severity of insulin-resistant diabetes in chronic patients. These epigenetic changes can be determined in the serum samples of diabetic patients. The epigenetic changes can be determined from $20 \mu \mathrm{l}$ serum samples by performing hydroxymethyl cytosine ELISA and data can be normalized by microtiter protein determination. The regression analysis can be performed to establish a correlation between hemoglobin glycosylation and epigenetic changes. Antidiabetic drugs such as Pioglitazone inhibit CB formation by serving as anti-apoptotic agents. Consequently, various anti-diabetic drugs are also used for the clinical management of AD. These antidiabetic drugs inhibit $\mathrm{CB}$ formation and prevent progressive neurodegeneration in the diabetes, AD and aging brain. Recently we reported that MTs inhibit CB formation by serving as free radical scavengers. CBs are pleomorphic pre-apoptotic biomarkers of compromised mitochondrial bioenergetics and are involved in progressive neurodegeneration in $\mathrm{AD}$ as Figure 2 illustrates.

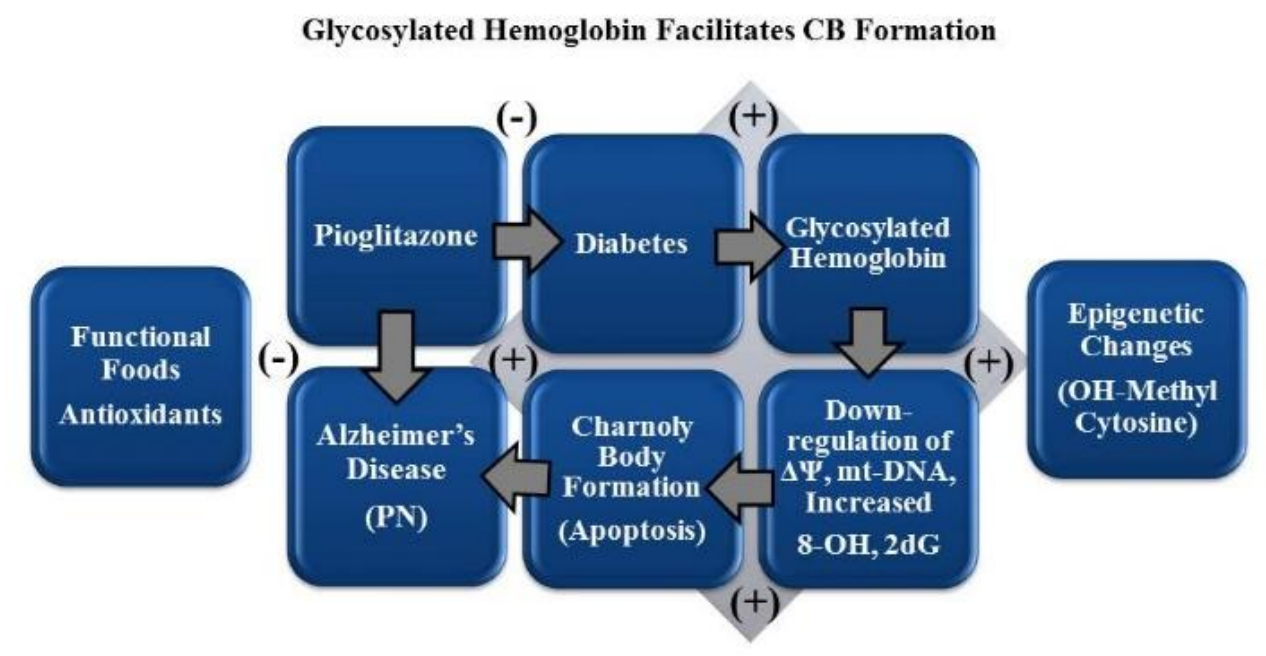

Figure 2. A schematic flow diagram illustrating that chronic diabetes induces glycosylation of hemoglobin. Its concentration is increased as a function of severity of illness and is reduced with antidiabetic treatment. Glycosylated hemoglobin compromises the mitochondrial bioenergetics by downregulating the $\Delta \Psi$ and mt-DNA, and increased synthesis of oxidation product, 8-OH, $2 \mathrm{dG}$, resulting in $\mathrm{CB}$ formation and apoptosis. These deleterious changes due to free radical overproduction cause progressive neurodegeneration in AD. Functional foods (rich in antioxidants i.e Resveratrol, Lycopenes, Catchsin, Sirtuins, Rutins, Flavinoids, Polyphenols) and anti-diabetic drugs such as Pioglitazone prevent $\mathrm{CB}$ formation and hence provide therapeutic benefit in diabetes as well as AD. 
Further studies in this direction will establish the exact functional significance of microRNAs and their impairments as a consequence of the epigenetic changes in diabetes. This study can establish the clinical significance of epigenetic changes in diabetes and their association with the severity of disease based on the extent of glycosylation of hemoglobin and clinical symptoms to provide an indirect estimate of glycemic index in the diabetic patients. Thus, biomarkers and drug targets may be developed to regulate DNA methylation to prevent and/or treat insulin-resistant diabetes as well as AD. Furthermore, this study can be extended to establish the exact cellular, molecular and genetic mechanism of epigenetic changes in IDDM and NIDDM patients and their precise relationship with DNA methylation and CB formation to induce progressive neurodegeneration in $\mathrm{AD}$. This study can provide future guidelines to establish evidence-based personalized theranostics (EBPT) of AD with disease-specific DNA methylation inhibitors [18].

\section{Autophagy-Related AD Therapies}

Mitochondrial dysfunction has an early and pivotal role in AD [61]. Mitochondria play a pivotal role in cell survival or demise because they are regulators of both energy metabolism as well as cell death pathways. Extensive literature supports a role for mitochondrial dysfunction and oxidative damage in the pathogenesis of AD. These investigators discussed the relationship between mitochondrial dysfunction and autophagy in $\mathrm{AD}$ and proposed that due to insufficient digestion of oxidatively-damaged macromolecules and organelles by autophagy, neurons accumulate lipofuscin that exacerbate neuronal dysfunction. Since autophagy is the major pathway involved in the degradation of protein aggregates and defective organelles including CBs as we have now discussed; interest in developing autophagy-related therapies has emerged. Hence autophagy is now considered a potential target of therapeutic interventions in AD pathophysiology. We have now proposed the development of novel charnolophagy agents in the early prevention and/or treatment of $\mathrm{AD}$. Whether antioxidants will facilitate or hinder in the therapeutic regimen of $\mathrm{AD}$ remains to be further established as some investigators believe that antioxidants may inhibit charnolophagy as a basic molecular mechanism of intracellular detoxification in progressive neurodegenerative diseases with increased incidence of either aggregated proteins or defective/degenerated organelle, including mitochondria primarily involved in $\mathrm{CB}$ formation during free radical-induced oxidative and nitrative stress of aging and $\mathrm{AD}$.

\section{MTs as CB Antagonists in Obesity}

MTs store, donate, buffer, and/or sequester zinc and are 30 times more potent as compared to glutathione in the mitochondria and nucleus. Hence therapeutic intervention inducing MTs would inhibit $\mathrm{CB}$ formation to provide neuroprotection in progressive neurodegenerative diseases such as $\mathrm{PD}, \mathrm{AD}$, MDD, schizophrenia, and multiple drug abuse. $\mathrm{CB}$ pathogenesis in the developing and/or aging brain supports the mitochondrial hypothesis of progressive neurodegeneration as we have now proposed. Hence drugs may be developed to prevent and/or inhibit CB formation, enhance charnolophagy, and/or inhibit CB sequestration in the developing and aging brain to cure obesity, diabetes, $\mathrm{AD}$, and other neurodegenerative diseases of unknown etiopathogenesis. We reported that nutritional and/or environmental oxidative and nitrative 
stress-induced free radical over-production augments CB formation in highly susceptible cell, whereas MTs inhibit CB formation by serving as free radical scavengers. In MTs gene manipulated mice we confirmed that MTs serve as CB antagonists to prevent obesity. MT $_{\mathrm{dko}}$ mice were mildly obese and lethargic, whereas $\mathrm{MT}_{\text {trans }}$ mice were lean and agile [17]. Hence physiological and/or pharmacological interventions to enhance MTs may prevent or inhibit obesity as illustrated in Figure 3.

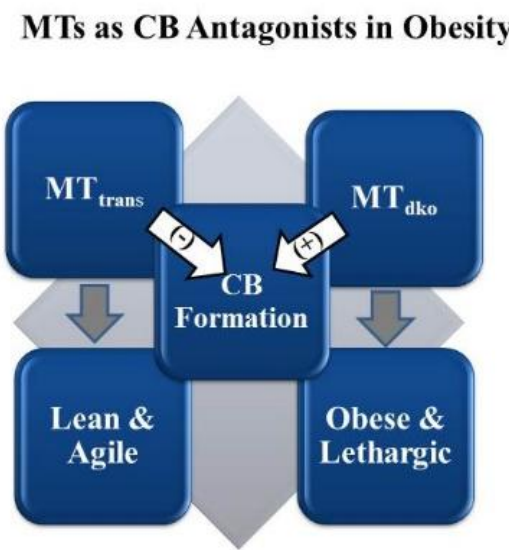

Fig. 3 A flow diagram illustrating the therapeutic potential of MTs as CB antagonists in obesity. $\mathrm{MT}_{\text {trans }}$ mice were lean and agile, whereas $\mathrm{MT}_{\mathrm{dko}}$ mice were obese and lethargic. $\mathrm{MT}_{\mathrm{dko}}$ mice were also highly susceptible to 1-Methyl, 4-Phenyl, 12.3,6 Tetrahydropyridine (MPTP)-induced Parkinsonism. (+) Activation; (-) Inhibition [17].

\section{Therapeutic Potential of MTs in AD}

There is now considerable evidence to support that mitochondrial damage plays a significant role in AD. Increased ROS generation and mitochondrial dynamic imbalance are suggested to be significant factors, as well as the consequence of Alzheimer-related pathology. Mitochondrial damage is related to $A \beta$ or tau pathology or to the presence of specific presenilin- 1 mutations [62]. We emphasized the key role of $\mathrm{CB}$ formation in the etiopathogenesis of $\mathrm{AD}$ as a consequence of compromised mitochondrial bioenergetics due to nutritional and environmental toxins-induced free radical overproduction and its attenuation by low molecular weight zinc binding proteins, MTs, which also provide neuroprotection by regulating the zinc-mediated transcriptional activation of genes involved in growth, proliferation, differentiation, and development. Due to the relevance of mitochondrial alterations in AD, recent works suggested the therapeutic potential of mitochondrially-targeted antioxidants. On the other hand, charnolophagy plays a significant role in intracellular detoxification as described above.

\section{TRPC-1-Mediated Neuroprotection}

It is well established that mammalian homologues of the Drosophila canonical transient receptor potential (TRP) proteins function as plasma membrane $\mathrm{Ca}^{2+}$ channels. We explored the neuroprotective role of TRPC1 in cultured SH-SY5Y cells treated with Parkinsonian neurotoxin, 1-methyl-4-phenylpyridinium ion (MPP(+), which significantly decreased TRPC1 on the plasma membranes [63]. TRPC1 over-expression reduced $\mathrm{MPP}^{+}$-induced neurotoxicity by inhibiting $\alpha$ - 
synuclein expression, whereas the over-expression of antisense TRPC1 cDNA construct or a nonspecific transient receptor potential channel blocker Lanthanum enhanced $\mathrm{MPP}^{+}$ neurotoxicity. Thapsigargine or carbachol-induced TRPC1 decreased $\mathrm{MPP}^{+}$neurotoxicity, which was partially dependent on $\left[\mathrm{Ca}^{2+}\right]_{\mathrm{ex}}$. Fluorescent staining of cells with an apoptotic biomarker (YO-PRO-1) demonstrated that TRPC1 protects SH-SY5Y cells against MPP ${ }^{+}$apoptosis. TRPC1 overexpression attenuated cytochrome c release and decreased Bax and Apaf-1 expression, suggesting that down-regulation of TRPC1 in response to $\mathrm{MPP}^{+}$may be involved in neurodegeneration. Thus, TRPC1 may be used as a sensitive biomarker in the inhibition of degenerative apoptotic signaling to provide neuroprotection against PD-inducing agents. However, the exact role TRCP1 in CB prevention and/or inhibition is yet to be established.

\section{Clinical Significance of Disease-Specific CBs in AD and other Diseases}

Various antipsychotic drugs such as Chlorpromazine, Chlorprazine, Risperidone, Domeperidone, are generally anti-dopaminergic as these are dopamine D-2 receptor antagonists. These typical, first generation, antipsychotic drugs alleviate positive symptoms of schizophrenia as compared to next generation, atypical antipsychotic drugs, as Quetiapine, Olanzapine, Clozapine, which alleviate the negative symptoms of schizophrenia and act on dopamine D3 and D4 receptors preferentially. The atypical antipsychotic drugs do not induce extrapyramidal symptoms as typical antipsychotic drugs, but can cause agranulocytosis; hence periodic blood analysis is required. These drugs can also induce hepatotoxicity, hypertension, hyperglycemia, obesity, and diabetes due to the down-regulation of MTs. We reported that MTs serve as CB antagonists to prevent obesity. Although $\mathrm{MT}_{\mathrm{dko}}$ mice did not exhibit any overt clinical symptoms of Parkinsonism, these genotypes are mildly obese and lethargic as compared $\mathrm{MT}_{\text {trans }}$ mice which are lean, thin, and agile. Chronic administration of MPTP induced severe body tremors, muscular rigidity, and complete immobilization in $\mathrm{MT}_{\mathrm{dko}}$ mice as compared to $\mathrm{MT}_{\text {trans }}$ mice which could still walk with their stiff legs and erect tail, suggesting the neuroprotective role of MTs in PD. MTs also inhibit $\mathrm{CB}$ formation by acting as free radical scavengers, whereas free radical overproduction augments $\mathrm{CB}$ formation in the most vulnerable aging cells in response to exposure to environmental neurotoxins such as Kainic acid and Domoic acid [60,61]. Chronic abuse of antipsychotic drugs can induce Parkinsonism associated with extrapyramidal symptoms. Although synaptic changes and adverse effects may take only hours - these drugs require minimum 2-3 weeks to have their obvious clinical effect. Chronic use of these drugs can also induce reversible MOA-B specific CB formation to cause Parkinsonism associated with extrapyramidal symptoms, whereas discontinuation of these drugs eliminates these adverse symptoms due to efficient MAO-B-specific CB eradication through Charnolophagy as a basic molecular mechanism of intracellular detoxification.

About $70-80 \%$ chronic drug addicts become victims to psychosis associated with schizophrenia due to excess and uncontrolled DA-ergic neurotransmission. Consequently, antiDA-ergic drugs alleviate the most distressing symptoms of schizophrenia to a certain extent yet with adverse extrapyramidal symptoms, whereas, drugs which enhance DA-ergic neurotransmission and/or dopaminergic agonists are used for the treatment of PD. Chronic abuse of these drugs induces hyper-sexuality and aggravate symptoms of schizophrenia in PD patients due to reversible MAO-A specific $\mathrm{CB}$ formation in the mesolimbic DAergic system. The 
symptoms of schizophrenia can be alleviated when these drugs are discontinued. Hence diseasespecific CBs can be used as novel targets for the future development of antipsychotic, antiAlzheimer, anti-parkinsonian, and antidepressant drugs with minimum or no adverse effects as experienced with currently available drugs in the market. Similarly, free radical-induced CB formation triggers intracellular $\mathrm{Ca}^{2+}$ and cholesterol crystallization due to $\Delta \Psi$ collapse, mitochondrial membranes degeneration, and TSPO delocalization in the adipocytes to cause atherosclerotic plaque formation, which is prevented by voltage-operated calcium channel blockers such as Verapamil, Nimodipine, and Diltiazem, whereas cholesterol crystallization is prevented by statins such as Simvastatin, Pitavastatin, Atrovastatin. Although these pharmaceutical agents have shown great promise as antihypertensive and anti-hypercholesterolemic drugs in obesity, their chronic use is associated with serious side effects. In contrast, ROS scavenging antioxidants such as Resveratrol, Polyphenols, Lycopenes, Catchsin, Sirtuins, Rutuins, and several LSDs derived from natural functional foods, can easily pass through blood brain barrier without any serious adverse side effects; thus, they can be used for the prevention and/or inhibition of $\mathrm{CB}$ formation involved in compromised mitochondrial bioenergetics and typical clinical manifestations. These antioxidants also have anti-apoptotic and anti-inflammatory properties to protect CNS and prevent and/or inhibit progressive neurodegeneration in PD, AD, ALS, HD, MS, chronic drug addiction, schizophrenia, and cardiovascular diseases of unknown etiopathogenesis. For example, antipsychotic drugs induce Parkinsonism, whereas various anti-Parkinson's drugs induce schizophrenia on chronic use due to $\mathrm{CB}$ formation to cause progressive neurodegeneration. Natural antioxidants derived from functional foods can inhibit $\mathrm{CB}$ formation by acting as free radical scavengers to prevent progressive neurodegeneration in $\mathrm{AD}$ and several other diseases as illustrated in Figure 4.

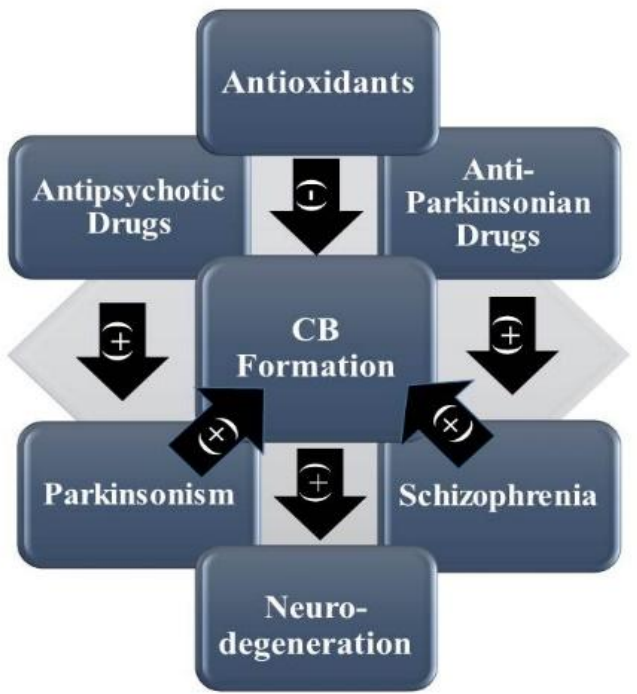

Figure 4. A diagram illustrating that antipsychotic drugs induce Parkinsonism, whereas various anti-Parkinson's drugs induce schizophrenia on chronic use due to CB formation to cause progressive neurodegeneration. Natural antioxidants derived from functional foods can inhibit $\mathrm{CB}$ formation by acting as free radical scavengers to prevent progressive neurodegeneration in $\mathrm{AD}$ and several other diseases. 


\section{Therapeutic Potential of MAO-A and MAO-B Specific CB Inhibitors}

There is limited information available regarding the exact therapeutic potential of specific MAO$\mathrm{A}$ and MAO-B specific $\mathrm{CB}$ inhibitors in $\mathrm{PD}, \mathrm{AD}$, drug addiction, $\mathrm{MDD}$, and schizophrenia. Recently, Sharma [19] proposed two different types of CBs depending on the type of MAO present on the outer mitochondrial membranes. MAO-As are localized primarily in the serotonergic and NE-ergic neurons in the limbic nervous system and are involved in pain, major depression, drug addiction, and schizophrenia, whereas MAO-Bs are localized in the nigrostriatal DA-ergic neuronal mitochondria. Thus, specific MAO-B inhibitors like Selegiline and Rasagiline provide neuroprotection in PD patients. A further study is needed to develop novel MAO-A and/or MAO-B specific CB inhibitors to explore their therapeutic potential in progressive neurodegenerative disorders such as $\mathrm{AD}$.

\section{Therapeutic Potential of Antioxidants as CB Inhibitors}

Recently we described in detail the therapeutic potential of various antioxidants in progressive neurodegenerative diseases and potential CB inhibitors [64]. These antioxidants are Resveratrol, Lycopene, Catchsin, Polyphenols, Flavonoids, Sirtuins, Rutins, which can be derived from natural sources and from functional foods. Although wine has an important antioxidant, Resveratrol $(250 \mu \mathrm{g} / 120 \mathrm{ml})$, it is insufficient to fulfil the daily requirement of $250 \mathrm{mg}$, which is 1000 times as compared to wine. More over wine contains nitrosamines which can cause cancer. In order to meet the daily requirement of Resveratrol from just wine, we may have to consume as many as 250 bottles of $120 \mathrm{ml}$ of wine, which will indeed cause hepatotoxicity and neurotoxicity much earlier as compared to the beneficial effects of Resveratrol derived from the wine as an antioxidant. Thus, natural sources of Resveratrol from functional foods or eating fresh grapes instead of wine will be more beneficial as compared to wine as a source of Resveratrol as generally publicized by the wine manufacturers and misinterpreted by the consumers. Recently we reported that salivary BDNF levels are significantly depleted in depression and stress-induced cortisol release triggers hippocampal CB formation to cause memory impairments and PTSD [65]. Consequently, diseases-specific antioxidants may be explored from functional foods to alleviate diversified symptoms of mood disorders including depression, anxiety, and memory impairments in $\mathrm{AD}$ and various diseases beyond the scope of this chapter.

\section{Functional Foods as Potential CB Inhibitors}

There are several functional food rich in antioxidants and can be used as potential CB inhibitors and booster of the mitochondrial bioenergetics to have therapeutic potential in $\mathrm{AD}$ and other progressive neurodegenerative diseases. For further details, please refer to author's recently published book on personalized medicine [66].

\section{CONCLUSION}

The basic understanding of sporadic AD has been a major challenge. Unlike the familial forms of $\mathrm{AD}$, the genetic and environmental risks factors for sporadic $\mathrm{AD}$ are extensive. $\mathrm{AD}$ is the most common form of dementia but the identification of reliable, early and non-invasive biomarkers remains a major challenge. We have proposed novel CB-based molecular biomarkers for detecting $\mathrm{AD}$ from peripheral cells including: skin cells, buccal mucosa cells, platelets, and 
lymphocytes and highlighted that compromised mitochondrial bioenergetics triggers $\mathrm{CB}$ formation involved in AD, PD, Pick's Disease. Alcoholism, Schizophrenia, chronic drug addiction (Cocaine, and METH), Down's syndrome, autism, Prion's disease, HD, ALS, MS, AIDs dementia, and several other chronic conditions of unknown etiopathogenesis. Nutritional stress, environmental toxins, and microbes can induce hippocampal atrophy, callosal atrophy, fronto-temporal cortical atrophy, ventriculomegaly, cerebral hyperthermia, dehydration, cerebral ischemia, stroke, and epilepsy due to free radical-induced $\mathrm{CB}$ formation, which can be ameliorated to a certain extent by supplementation of antioxidant-rich functional foods in old age to prevent progressive neurodegeneration, cognitive impairment, and dementia in $\mathrm{AD}$ and other neurodegenerative diseases. The basic advantage of antioxidants derived from functional foods (including Resveratrol, Lycopenes, Sirtuins, Rutins, Catschin, Flavonoids, and Polyphenols) is that these can pass easily through blood brain barrier and do not produce adverse side effects as noticed in other standard pharmaceutical agents. Presently, we have limited scope of removing any normal or pathological brain region because of its limited regenerative potential and serious impairment in the sensory modality due to somatotropic representation. Consequently, the therapeutic benefit of antioxidants is highly significant. However, the main limitation of antioxidants derived from functional foods and Mediterranean diets is that they have reduced potency. As a result, their therapeutic potential is also limited at this moment. Serious attempts are being made to develop specific antioxidant-loaded ROS scavenging nanoparticles to enhance their adequate delivery in the CNS and other organs for AD prevention and treatment.

List of Abbreviations: Alzheimer's disease, AD; Amyotropic lateral sclerosis, ALS;

BDNF: Brain Derived Neurotropic Factors, BDNF; Charnoly Body, CB; 18F-Dihydroxyphenyl alanine, ${ }^{18}$ D-DOPA; ${ }^{18}$ F-Fluorodeoxyglucose, ${ }^{18} \mathrm{FdG}$; 8-Hydroxy Guanosine, 8-OH; 2 deoxy Guanosine, 2dG; Fetal Alcohol Syndrome, FAS; Gastrointestinal Tract, GIT; Huntington's disease, HD; Insulin Like Growth Factor-1, IGF-1; Monoamine Oxidase-A, MAO-A; Monoamine Oxidase-B, MAO-B; Multiple Sclerosis, MS; Metallothioneins, MTs; Parkinson's disease, $\mathrm{PD}$; $\mathrm{RhO}_{\mathrm{mgko}}$ : Mitochondrial Genome Knock out; SI: Synuclein Index (A ratio of nitrative synuclein vs native synuclein); Synuclein-Metallothioneins Triple Knock Out, Syn$\mathrm{MT}_{\text {tko }}$; Homozygous weaver Mutant Mice, wv/wv Mice; MTs over-expressed weaver mice, wv/wv-MTs

Competing Interests: There are no competing interests in this chapter.

Author's Contribution: This chapter written based on Professor Sushil Sharma's several years of original research conducted in India, Canada, and U.S.A. The proposed hypothesis is being further explored in collaborative research work with co-authors from the Saint James School of Medicine, St Vincent. The following M.D students are conducting research with Prof. Sharma on topics relevant to subject matter of this chapter. All of these students have thoroughly read, edited, and contributed in the recent literature search for this chapter.

Acknowledgement and Funding: The authors express sincere thanks to President Kallol Guha, President Saint James Schools of Medicine and all faculty members for their moral support and 
encouragement. Professor Sharma conducted several experiments in collaboration with Ex Vice President Manuchair Ebadi, University of North Dakota School of Medicine, Center of Excellence in Neurosciences, Grand Forks, ND, U.S.A. Emeritus Professor K. Dakshinamurti, University of Manitoba School of Medicine, Department of Biochemistry and Molecular Biology, Winnipeg, Manitoba (Canada) and late Emeritus Professor Baldev Singh, All India Institute of Medical Sciences, New Delhi (India) are also acknowledged for their constant encouragement and moral support. This research was conducted in several labs in India, Canada, and U.S.A and was financially supported by various multinational funding agencies.

\section{REFERENCES:}

1. Sharma, S. K., Nayar, U., Maheshwari, M.C, and Gopinath, G. Ultrastructural studies of P-cell morphology in developing normal and undernourished rat cerebellar cortex. Electrophysiological Correlates. Neurology. India. 34: 323-327, 1986.

2. Sharma, S. K., Nayar, U., Maheshwari, M.C. and Singh, B. Effect of undernutrition on developing rat cerebellum: Some Electrophysiological and Neuromorphological Correlates. J. Neurol. Sciences. 78: 261-272, 1987.

3. Sharma, S.K., Selvamurthy, W. and Dakshinamurti, K. Effect of environmental neurotoxins in the developing brain. Biometeorology. 2: 447-455. 1993.

4. Dakshinamurti, K., Sharma, S.K., Sundaram, M., and Watanabe T. Hippocampal.changes in developing postnatal Mice following Intra-uterine Exposure to Domoic acid. J. Neurosci. 13: 4486-4495, 1993

5. Sharma, S.K., and Dakshinamurti, K. Suppression of Domoic acid-induced seizures by 8-(OH)-DPAT. J. Neural Transmission.93: 87-89, 1993.

6. Sharma S, Moon CS, Khogali A, Haidous A, Chabenne A, Ojo C, Jelebinkov M, Kurdi Y, Ebadi M. Biomarkers of Parkinson's Disease (Recent Update). Neurochemistry International. 63:201-229. 2013.

7. Sharma S, Rais A, Sandhu R, Nel W, Ebadi M: Clinical significance of metallothioneins in cell therapy and nanomedicine. International Journal of Nanomedicine. 8: 1477-1488. 2013.

8. Jagtap A, Gawande S, \& Sharma S. Biomarkers in Vascular Dementia 7: 43-56. 2014.

9. Sharma S, Gawande S, Jagtap A, Abeulela R, and Salman Z. Fetal Alcohol Syndrome; Prevention, Diagnosis, \& Treatment. In Alcohol Abuse: Prevalence, Risk Factors. Nova Science Publishers, New York, U.S.A; 2015 Chapter 3, pp 39-94.

10. Chabenne A, Moon C, Ojo C, Khogali A, Nepal B, and Sharma S. Biomarkers in Fetal Alcohol Syndrome (Recent Update) Biomarkers and Genomic Medicine 6:1222, 2014.

11. Sharma S, Nepal B, Moon CS, Chabenne A, Khogali A, Ojo C, Hong E, Goudet R, Sayed-Ahmad A, Jacob A, Murtaba M, Firlit M. Psychology of Craving. Open Jr of Medical Psychology. 3, 120-125, 2014.

12. Sharma S, Ebadi M: Therapeutic Potential of Metallothioneins as Anti-inflammatory Agents in Polysubstance Abuse. Journal of Institute of Integrative Omics \& Applied Biotechnonogy IIOAB Journal 2:50-61, 2011. 
13. Sharma S. Charnoly body as a universal biomarker of multiple drug addiction, $3^{\text {rd }}$ International Conference on drug Addiction, Orlando, FL, U.S.A. Aug 3-5, 2015) [Invited Speaker and Chairperson].

14. Sharma S, Ebadi M: Therapeutic Potential of Metallothioneins in Parkinson's disease. In New Research on Parkinson's Disease. Eds T.M Hahn and Julian Werner: Nova Science Publishers, New York, U.S.A. 1-41, 2008.

15. Sharma S, Ebadi M: Metallothioneins As Early \& Sensitive Biomarkers of Redox Signaling in Neurodegenerative Disorders. Journal of Institute of Integrative Omics \& Applied Biotechnonogy (IIOAB Journal) 2:98-106, 2011.

16. Sharma $S$ and Ebadi $M$. Significance of Metallothioneins in Aging Brain. Neurochemistry International. 65: 40-48, 2014.

17. Sharma S., Kheradpezhou M., Shavali S., EI Refaey H., Eken J., Hagen C., \& Ebadi M. (2004) Neuroprotective Actions of Coenzyme $Q_{10}$ in Parkinson's Disease. Methods in Enzymology. 382:488-509, 2004.

18. Sharma S. Nanotheranostics in Evidence Based Personalized Medicine. Current Drug Targets. 15: 915-930, 2014.

19. Sharma S (ed): Monoamine Oxidase Inhibitors: Clinical Pharmacology, Benefits, and Potential Health Risks. Nova Science Publishers, New York, U.S.A. 2016.

20. Sharma S, Carlson E, and Ebadi M. The Neuroprotective Actions of Selegiline in Inhibiting 1-Methyl, 4-Phenyl, Pyridinium Ion (MPP+)-Induced Apoptosis in Dopaminergic Neurons. J. Neurocytology 32: 329-343, 2003.

21. Sharma S, Ebadi M: Charnoly body as a Universal Biomarker of Cell Injury. Biomarkers and Genomic Medicine. 6: 89-98, 2014.

22. Sharma S. Ebadi M: In-Vivo Molecular Imaging in Parkinson's Disease. In Parkinson's Disease. Eds. RF. Pfeiffer, ZK Wszolek, M. Ebadi. Ind Edition, CRC Press Taylor \& Francis Group. Boca Rotan, FL, U.S.A. Chapter 58, 787-790. 2013.

23. Sharma S. and Ebadi M. Distribution Kinetics of ${ }^{18}$ F-DOPA in Weaver Mutant Mice. Molecular Brain Research 139:23-30, 2005.

24. Sharma S, Refaey H. El, \& Ebadi M. Complex-1 activity and ${ }^{18}$ F-DOPA uptake in genetically engineered mouse model of Parkinson's disease and the neuroprotective role of coenzyme $\mathrm{Q}_{10}$. Brain Res. Bull. 70: 22-32, 2006.

25. Ebadi M. \& Sharma S. Metallothioneins 1 and 2 Attenuate PeroxynitriteInduced Oxidative Stress in Parkinson's disease. Exp. Biol. Med. 231:1576-1583, 2006.

26. Sharma S, Ebadi M: SPECT Neuroimaging in Translational Research of CNS Disorders. Neurochem Internat. 52:352-362, 2008.

27. Henchcliffe C: Blood and cerebrospinal fluid markers in Parkinson's disease: current biomarker findings. Current Biomarker Findings. 5: 1-11, 2015.

28. Zhang S, Lei C, Liub P, Zhang M, Taoa W, Liud H, Liu, M: Association between variant amyloid deposits and motor deficits in FAD-associated presenilin-1 mutations: A systematic review. Neuroscience and Biobehavioral Reviews 56: 180192, 2015.

29. Li Z, Lin Q, Ma Q, Lu C, Tzeng CM: Genetic predisposition to Parkinson's disease and cancer. Curr Cancer Drug Targets. 14: 310-321, 2014. 
30. Vyas S, Rodrigues AJ, Silva JM, Tronche F, Almeida OFX, Sousa N, and Sotiropoulos I. Chronic Stress and Glucocorticoids: From Neuronal Plasticity to Neurodegeneration. Neural Plasticity. 2016: 1-15, 2016.

31. de Pablos RM, Herrera AJ, Espinosa-Oliva AM, Sarmiento M, Muñoz MF, Machado A, Venero JL. Chronic stress enhances microglia activation and exacerbates death of nigral dopaminergic neurons under conditions of inflammation. Journal of Neuroinflammation 11:34, 2014.

32. Herrera AJ, Espinosa-Oliva AM, Carrillo-Jimenez A, Oliva-Martin MJ, GarciaRevial J, Garcia-Quintanilla A, de Pablos RM, Veneri JL. Relevance of chronic stress and the two faces of microglia in Parkinson's disease. Front Cell Neurosci. 9: 312, 2015.

33. Herrero MT, Estrada C, Maatouk L, Vyas S. Inflammation in Parkinson's disease: role of glucocorticoids. Front Neuroanat. 9: 32, 2015.

34. Sharma S. (ed): 2014. Beyond Diet and Depression (Volume-1) Book Nova Sciences Publishers, New York; 2014.

35. Sharma S: Beyond Diet and Depression (Volume-2) Book Nova Science Publishers, New York; 2014.

36. Sharma, S (ed): Alleviating Stress of the Soldier and Civilian. Nova Science Publishers, New York; 2015.

37. Sharma SK, Ebadi M: An improved method for analyzing coenzyme Q homologues and multiple detection of rare biological samples. J Neurosci Methods. 137:1-8, 2004.

38. Ebadi M, Sharma SK, Wanpen S, Amornpan A: Coenzyme $Q_{10}$ inhibits mitochondrial complex-1 down-regulation and nuclear factor-kappa B activation. J Cell Mol Med. 8(2):213-222, 2004.

39. Dakshinamurti K, Sharma SK, Geiger JD: Neuroprotective actions of pyridoxine. Biochim Biophys Acta. 1647:225-229, 2003.

40. Lal KJ, Sharma SK, Dakshinamurti K: Regulation of calcium influx into vascular smooth muscle by vitamin B6. Clin Exp Hypertens. 15:489-500, 1993.

41. Dakshinamurti S, Dakshinamurti K: Antihypertensive and neuroprotective actions of pyridoxine and its derivatives. Can J Physiol Pharmacol. 93:1083-1090, 2015.

42. Ebadi M, Sharma S, Shavali S, El Refaey H. Neuroprotective actions of selegiline. J Neurosci Res. 67(3):285-289, 2002.

43. Ebadi M, Brown-Borg H, Sharma S, Shavali S, El ReFaey H, Carlson EC: Therapeutic Efficacy of Selegiline in Neurodegenerative Disorders and Neurological Diseases. Current Drug Targets. 7: 1-17. 2006.

44. Sharma, S.K., and Ebadi, M. Metallothionein Attenuates 3Morpoholinosydnonimone (SIN-1)-Induced Oxidative and Nitrative Stress in Dopaminergic Neurons. Antioxidants and Redox Signaling. 5: 251-264, 2003.

45. Ebadi, M., and Sharma S. Peroxynitrite and Mitochondrial Dysfunction in the Pathogenesis of Parkinson's disease. Antioxidants \& Redox Signaling 5: 319-335. 2003. 
46. Sharma S: Progress in PET Radiopharmaceuticals (Quality Control \& Theranosics). Nova Science Publihsers, New York, US.A.

47. Hargreaves RJ, Rabiner EA: Translational PET imaging research. Neurobiol Dis. 61:32-38, 2014.

48. Sharma S. Charnoly Body as a Novel Biomarker in Zika Virus-Induced Microcephaly. Drug Discovery and Therapy World Congress, Boston, Mass, U.S.A. Aug 22-25, 2016 (Invited Lecture).

49. Maes OC, Chertkow HM, Wang E, Schipper HM: MicroRNA: Implications for Alzheimer Disease and other Human CNS Disorders. Curr Genomics. 10(3): 154$168,2009$.

50. Schonrock N, Matamales M, Ittner LM, Götz J: MicroRNA networks surrounding APP and amyloid- $\beta$ metabolism--implications for Alzheimer's disease. Exp Neurol. 235: 447-454, 2012.

51. Tomasetti M, Neuzil J, Dong L: MicroRNAs as regulators of mitochondrial function: Role in cancer suppression. Biochimica et Biophysica Acta (BBA) General Subjects. Frontiers of Mitochondrial Research. 1840: 1441-1453, 2014.

52. Duarte FV, Palmeira CM, Rolo AP: 2015. The Emerging Role of MitomiRs in the Pathophysiology of Human Disease. Adv Exp Med Biol. 888: 123-154, 2015.

53. Leidinger P, Backes C, Deutscher S, Schmitt K, Mueller SC, Frese K, Haas J, Ruprecht, K, Paul F et al.: A blood based 12-miRNA signature of Alzheimer disease patients. Genome Biology. 14: R78, 2013.

54. Lykhmus O, Mishra N, Koval L, Kalashnyk O, Gergalova G, Uspenska K, Komisarenko S, Soreq H, Skok M: Molecular Mechanisms Regulating LPSInduced Inflammation in the Brain. Front Mol Neurosci. 9: 19, 2016.

55. Shi Q, Gibson GE: Up-regulation of the mitochondrial malate dehydrogenase by oxidative stress is mediated by miR-743a. J. Neurochem. 118: 440-448, 2011.

56. Logue SE, Cleary P, Saveljeva S, Samali A: New directions in ER stress-induced cell death. Apoptosis. 18: 537-546, 2013.

57. DuBoff B, Götz J, Feany MB: Tau promotes neurodegeneration via DRP1 mislocalization in vivo. Neuron. 75(4):618-632, 2012.

58. Hayashi H, Nakagami H, Takeichi M, Shimamura M, Koibuchi N, Oiki E, Sato N, Koriyama H, Mori M, Gerardo Araujo R, Maeda A, Morishita R, Tamai K, Kaneda Y: HIG1, a novel regulator of mitochondrial $\gamma$-secretase, maintains normal mitochondrial function. FASEB J. 26:2306-2317, 2012.

59. Kandimalla R, Reddy PH: 2016. Multiple faces of dynamin-related protein 1 and its role in Alzheimer's disease pathogenesis. Biochim Biophys Acta. 1862: 814-828, 2016.

60. Chen Y, Parker WD, Chen H, Yang K: Aberrant mitochondrial RNA in the role of aging and aging associated diseases. Medical Hypotheses. 85: 178-182, 2013.

61. Moreira PI, Carvalho C, Zhu X, Smith MA, Perry G: Mitochondrial dysfunction is a trigger of Alzheimer's disease pathophysiology. Biochimica et Biophysica Acta (BBA) - Molecular Basis of Disease. 1802: 2-10, 2010. 
62. García-Escudero V, Martín-Maestro P, Perry G, and Avila J: Deconstructing Mitochondrial Dysfunction in Alzheimer Disease. Oxidative Medicine and Cellular Longevity. 1-13, 2013.

63. Bollimuntha S, Singh BB, Shavali S, Sharma SK, Ebadi M: TRPC1-mediated inhibition of 1-methyl-4-phenylpyridinium ion neurotoxicity in human SH-SY5Y neuroblastoma cells. J Biol Chem. 280:2132-2140, 2005.

64. Sharma S: Molecular Pharmacology of Environmental Neurotoxins. In Kainic Acid: Neurotoxic Properties, Biological Sources, and Clinical Applications. Nova Science Publishers. New York. P1-47, 2014.

65. Sharma S, Ebadi M: Antioxidant Targeting in Neurodegenerative Disorders. Ed. I. Laher, Springer Verlag. Germany. Chapter 85, p. 1-30, 2014.

66. Sharma S. Personalized Medicine (Beyond PET Biomarkers) Nova Science Publishers, New York, U.S.A. 2016. 